\title{
Effect of biofouling roughness on a marine propeller's performance including cavitation and underwater radiated noise (URN)
}

\author{
Savas Sezen*, Dogancan Uzun, Refik Ozyurt, Osman Turan, Mehmet Atlar \\ Department of Naval Architecture, Ocean \& Marine Engineering, University of \\ Strathclyde
}

\begin{abstract}
:
This study aims to investigate the effects of biofouling-related roughness on a propeller's hydrodynamic and underwater radiated noise (URN) performance. Selected benchmark INSEAN E779A propeller operated in uniform \& open water flow under non-cavitating and cavitating conditions. The hydrodynamic flow field around the propeller was first solved using RANS (Reynoldsaveraged Navier Stokes) solver. The Schnerr-Sauer cavitation model, based on reduced RayleighPlesset equation, was used to model the sheet cavitation on the propeller blades and tip vortex cavitation (TVC) in the propeller's slipstream. A vorticity-based Adaptive Mesh Refinement (AMR) technique was employed for the observation of TVC. The porous form of the Ffowcs-Williams Hawkings (P-FWH) equation, which is coupled with the RANS solver, was used to predict the URN (or hydroacoustic performance) of the propeller. The propeller performance characteristics, including cavitation, were validated with the available experimental data. Following that, the roughness functions representing the different roughness configurations obtained from the literature were employed using wall function model of Computational Fluid Dynamics (CFD) solver. The results showed that roughness has detrimental impacts on the propeller's performance characteristics. That is to say that the propeller's thrust decreases while the torque increases with increasing severity of the roughness. Hence, the efficiency loss of the propeller at the most severe roughness condition can be as high as $30 \%$ and $25 \%$ at $\mathrm{J}=0.795$ and $\mathrm{J}=0.71 \& \sigma=1.763$, respectively. Unlike its detrimental effects on the hydrodynamic performance, the roughness had some positive effects by reducing the cavitation volume, especially for the TVC and hence on the propeller underwater radiated noise (URN). The results also indicated that the URN levels might be reduced up to $10 \mathrm{~dB}$ between $1 \mathrm{kHz}$ and $2 \mathrm{kHz}$. Besides, $2^{\text {nd }}$ and $3^{\text {rd }}$ BPF values decrease between 1 and $7 \mathrm{~dB}$ under varying roughness configurations in comparison to the smooth case. The study reported the effect of a particular biofouling roughness on the URN levels of a propeller for the first time in model-scale and using the CFD simulations.
\end{abstract}

Keywords: Roughness, Cavitation, Tip Vortex Cavitation, Mitigation, Marine Propeller, URN, hydroacoustic, FW-H. 


\section{NOMENCLATURE}

\begin{tabular}{|c|c|c|c|}
\hline URN & Underwater Radiated Noise & $\mathrm{CPU}$ & Central Processing Unit \\
\hline AMR & Adaptive Mesh Refinement & $\mathrm{GCl}$ & Grid Convergence Index \\
\hline P-FWH & $\begin{array}{l}\text { Porous Form of the Ffowcs-Williams } \\
\text { Hawkings }\end{array}$ & $\mathrm{k}$ & Turbulence Kinetic Energy $\left(\mathrm{m}^{2} / \mathrm{s}^{2}\right)$ \\
\hline CFD & Computational Fluid Dynamics & $\omega$ & Turbulence Dissipation (1/s) \\
\hline TVC & Tip Vortex Cavitation & $\rho$ & Density $\left(\mathrm{kg} / \mathrm{m}^{3}\right)$ \\
\hline IMO & International Maritime Organisation & $\mu$ & Dynamic Viscosity (Pa.s) \\
\hline ABS & American Bureau of Shipping & $u$ & Velocity $(\mathrm{m} / \mathrm{s})$ \\
\hline ITTC & International Towing Tank Conference & $T_{R e}$ & Reynolds Stress Tensor (N) \\
\hline GHG & Greenhouse Gas & $F$ & Additional Momentum Sources (N) \\
\hline DNV-GL & Det Norske Veritas- Germanisher Loyd & $\alpha$ & Gas Volume Fraction \\
\hline RINA & Royal Institution of Naval Architects & $\dot{m}$ & $\begin{array}{l}\text { Mass Flow Rate per Unit Volume } \\
(\mathrm{kg} / \mathrm{s})\end{array}$ \\
\hline EU & European Union & $R$ & Local Bubble Radius (m) \\
\hline RANS & Reynolds-averaged Navier-stokes & $n_{0}$ & $\begin{array}{l}\text { Number of Seeds per Unit Volume of } \\
\text { Liquid }\end{array}$ \\
\hline DES & Detached Eddy Simulation & $U$ & Modified Velocity $(\mathrm{m} / \mathrm{s})$ \\
\hline LES & Large Eddy Simulation & $L$ & Modified Stress Tensor (N) \\
\hline PPTC & Potsdam Propeller Test Case & $u_{i}$ & Fluid Velocity $(\mathrm{m} / \mathrm{s})$ \\
\hline SST & Shear Stress Transport & $v_{i}$ & Surface Velocity $(\mathrm{m} / \mathrm{s})$ \\
\hline DSM & Density Corrected Model & $P_{i j}$ & Compressive Stress Tensor (N) \\
\hline VOF & Volume of Fluid & $\delta$ & Kronecker Symbol \\
\hline DNS & Direct Numerical Simulations & $r$ & $\begin{array}{l}\text { Distance between the Noise Source } \\
\text { and Receiver }(\mathrm{m})\end{array}$ \\
\hline ow & Open Water & $c_{0}$ & Speed of Sound $(\mathrm{m} / \mathrm{s})$ \\
\hline BPF & Blade Passage Frequency & $p$ & Acoustic Pressure (Pa) \\
\hline KCS & Kriso Container Ship & $\kappa$ & Von Karman Constant \\
\hline TE & Trailing Edge & $y^{+}$ & $\begin{array}{l}\text { Non-Dimensional Normal Distance } \\
\text { from The Boundary }\end{array}$ \\
\hline LE & Leading Edge & $B$ & Smooth Log Law Intercept \\
\hline$S P L$ & Sound Pressure Level ( $d B)$ & $\Delta U^{+}$ & Roughness Function \\
\hline MRF & Moving Reference Frame & $U_{\tau}$ & Friction Velocity $(\mathrm{m} / \mathrm{s})$ \\
\hline
\end{tabular}




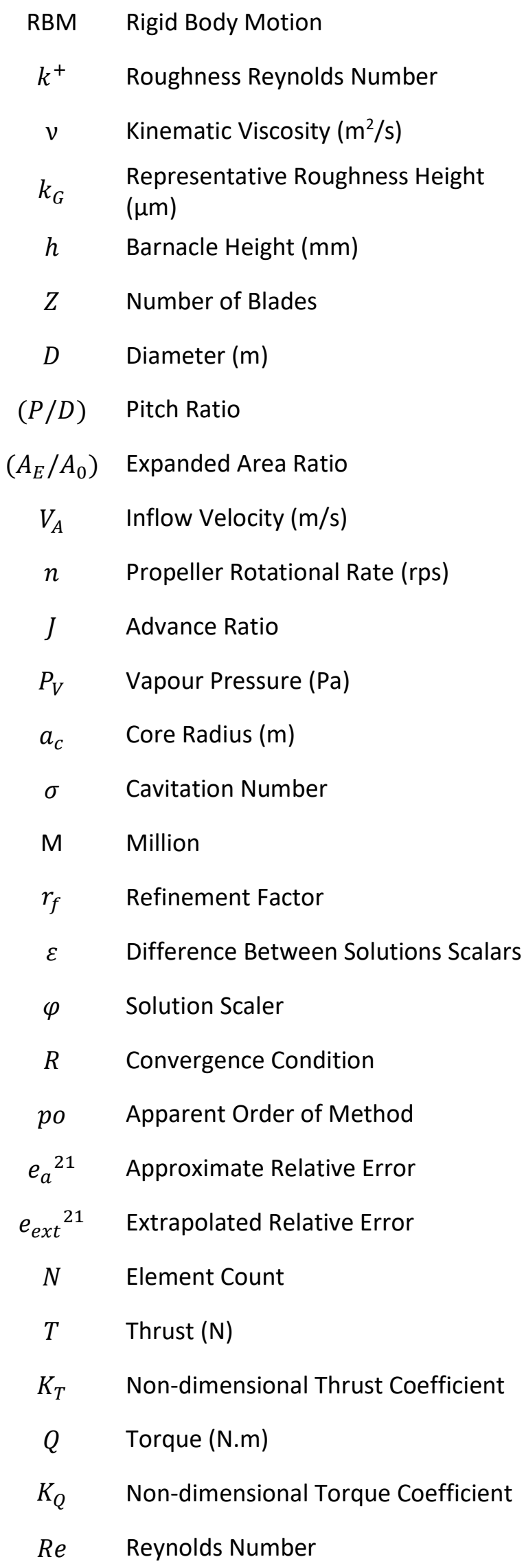




\section{INTRODUCTION}

Marine biofouling has been identified as one of the essential problems in both aspects of economic and ecologic by IMO. Biofouling causes degradation in hydrodynamic performance of ships by increasing the hull resistance, which in turn increases the fuel oil consumption and greenhouse gas (GHG) emissions.

Although the effects of biofouling roughness on the ship hull performance have been investigated profoundly in the literature [1], [2], [3], [4], [5], [6], [7], there is a lack of literature for the roughness effect on propeller hydrodynamic performance [8]. According to the American Bureau of Shipping (ABS) [9] report, fouling accumulates on a propeller within a year show a wide range of diversity including slime, algae and even barnacles and tube worms. Fouling accumulation over a year of operation on a propeller can be seen in Figure 1 as an example. The ITTC [10] suggested that new methods and strategies need to be developed to understand the effect of coatings and biofouling roughness on overall ship performance, including the propeller.

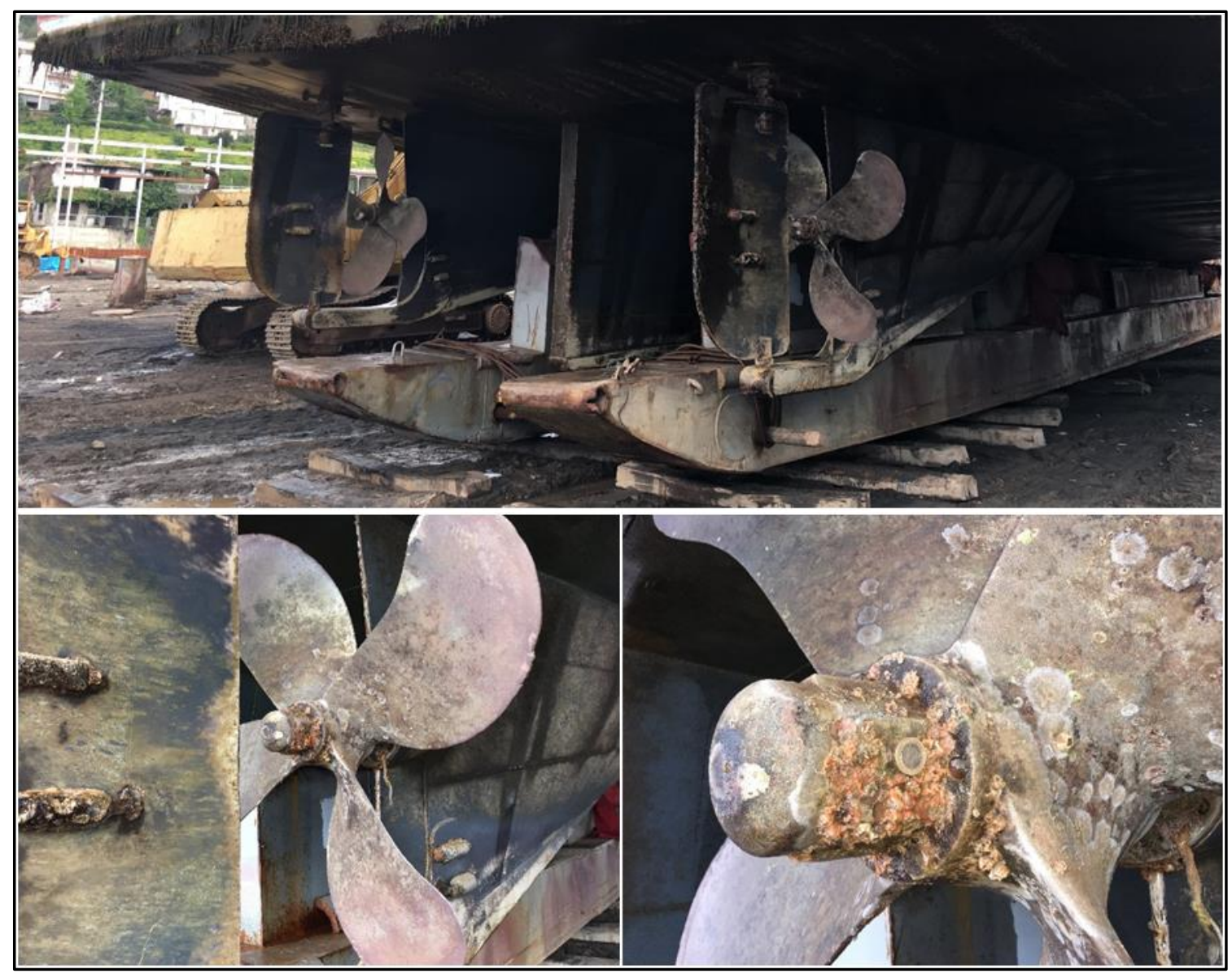

Figure 1 Biofouling after a year of operation on a propeller

While the concern is the efficiency and hence the fuel consumption for the shipping industry, the effects of roughness on the propeller cavitation and underwater radiated noise (URN) are also 
important for the naval vessels, passenger comfort in cruise vessels and the possible impact of on marine fauna.

The URN characteristics of naval vessels (e.g. submarines) have always been the primary concern for their designs and operations due to the strict requirements for the stealth mode of operations. However, recently, the attention has also moved towards the environmental impacts of the URN induced by commercial vessels [11] as a result of continually increasing volume of shipping traffic in the world's oceans. The increased URN levels of the commercial vessels, mainly associated with the cavitating propeller, have been considered as a major threat to the marine ecosystem, especially for the well-being of the marine animals in terms of their communication, navigation and hunting [12]; [13]. As a result, international associations and classification societies have included this issue in their agendas and advised the industry to take precautions to decrease its detrimental effects on marine fauna [14]. In this regard, the international non-mandatory rules such as Det Norske VeritasGermanisher Loyd (DNV-GL) QUIET class, Royal Institution of Naval Architects (RINA) DOLPHIN notations are becoming widespread to minimise the noise emission in the oceans. Moreover, IMO has also pointed out this issue as a high priority and published a non-mandatory guideline to reduce the URN mainly induced by commercial ships [15]. Consequently, several concerted efforts have been spent to measure, predict and mitigate the URN thereby aiming at $3 \mathrm{~dB}$ in 10 years and $10 \mathrm{~dB}$ in 30 years [16] through numerous collaborative EU and international projects, e.g. [17], [18], [19], [20], [21].

Limited numerical and experimental research studies have been conducted in the literature for the prediction of propeller hydrodynamic and hydroacoustic performance under non-cavitating and cavitating conditions. However, the effects of roughness on the propeller's hydrodynamic performance in the presence of sheet and tip vortex cavitation (TVC) have not been investigated adequately using CFD, and hence its impact on the URN characteristics of a propeller is totally unknown. Hence, this study aims to explore this gap by adopting a numerical method by using a stateof-the-art commercial CFD tool with a modified wall function approach using the experimental roughness data.

In this study, the initial step is to validate the propeller global performance characteristics (i.e. thrust, torque and efficiency) with the available experimental data under non-cavitating conditions for a wellknown benchmark propeller, INSEAN E779A. After that, the Schnerr-Sauer cavitation model, which is based on the reduced Rayleigh-Plesset equation, is employed to model the sheet and TVC. A vorticitybased Adaptive Mesh Refinement (AMR) technique is implemented for better modelling of the TVC in the propeller's slipstream. The hydrodynamic results, including cavity pattern, are validated with the experimental data. Following this, the hybrid method, which combines RANS and porous form of the 
Ffowcs Williams Hawkings (P-FWH) equation, is used to predict the hydroacoustic propeller performance. The numerical hydrodynamic and hydroacoustic pressures are compared with each other in the near field to show the reliability of the numerical solution. The numerical model is then modified to account for the roughness effects on the propeller's hydrodynamic performance under non-cavitating and cavitating conditions, and URN under cavitating conditions. The effect of the different roughness levels on the thrust, torque, efficiency, cavitation patterns and URN characteristics of the propeller for various operating conditions are explored, and results are discussed. The study explored the effect of a particular biofouling roughness on the URN levels of a propeller for the first time in model-scale and using the CFD simulations.

Within the above framework, the following section of the paper presents a literature survey associated with the limited investigations into the effect of roughness on the propeller performance, cavitation and URN characteristics. This is followed by the theoretical background of the numerical model used in this study, including fundamental equations for the hydrodynamic and hydroacoustic models as well as the roughness in Section 3. Section 4 presents the application details of the numerical modelling, including computational domain \& boundary conditions, grid structure with Adaptive Mesh Refinement (AMR) technique, and solution algorithm. The numerical results and discussions for the non-cavitating and cavitating conditions are given in Section 5. Finally, the conclusions are presented in Section 6.

\section{LITERATURE REVIEW}

This section provides a general literature review for the studies investigating the effects of biofouling on overall ship performance in the broad aspect, but with a keen interest in the propeller performance. There are many studies conducted in the literature focusing on the penalties caused by biofouling on ship hulls such as [1], [22], [23], [24], [25]. However, it is important to note that since it is a very distinctive subject, there are limited researches performed for investigating the effect of roughness on propeller performance, cavitation and noise.

Townsin et al. [26] drew attention on penalties caused by propeller roughness due to biofouling. Moreover, a cost-efficiency analysis was conducted in order to compare hull and propeller cleaning operations. Additionally, Mosaad [27] highlighted the energy loss per unit area due to the rough propeller condition and how important it is in comparison with the hull roughness. Atlar et al. [28] investigated the influence of foul release coatings on a propeller and showed that the propeller blade roughening causes significant penalties in ship performance due to the changes in torque and thrust produced by the propeller. In the following study, Atlar et al. [29] conducted a similar analysis showing the efficiency loss in higher speed vessels such as military craft. In their study, the analysis showed that preventing biofouling on propeller blades of high-speed vessels have a higher potential of 
increasing the efficiency than slower vessels propeller. As stated in the study of Atlar et al. [29], Anderson et al. [30] conducted a study and found that clean propeller has the favourable cost benefits compared to a roughened propeller. Seo et al. [31] developed an algorithm and investigated the impacts of biofouling on a propeller performance in open water. The authors ' results showed that biofouling present on the propeller increases the torque with increasing fouling rate and therefore, a loss of efficiency is experienced.

With an increasing interest in Computational Fluid Dynamics (CFD), Krüger et al. [32] investigated the effects of roughness on open water propeller performance under non-cavitating conditions using RANS and LES (Large Eddy Simulation) coupled with roughness model. The main aim of the study was to investigate the effects of propeller tip roughness and application area on the tip vortex pressure. First, the sand grain roughness and structured dimples were employed by modelling only one blade of the propeller. The results showed that suction-side roughness is the most effective solution to keep the propeller efficiency as high as possible by increasing the pressure inside the tip vortex. Owen et al. [8] investigated the impacts of fouling on the performance of a Potsdam Propeller Test Case (PPTC) by using CFD. In their study, the authors stated that their research results could be regarded as a proof for which the CFD is a very practical way to predict the penalties caused by fouling accumulation on a propeller based upon the numerical results. Asnaghi et al. [33] investigated the probability and effectiveness of varying roughness characteristics, such as the roughness height and roughness coverage area, to mitigate tip vortex flows using CFD for elliptical foil. This study demonstrated that cavitation inception could be delayed with the application of roughness. Another CFD based study was conducted by Song et al. [34]. In their study, Song et al. [34] investigated the deteriorating effects of biofouling on ship propeller by proposing a CFD model depending on Demirel et al. [6]. The very recent and comprehensive numerical and experimental study was conducted by Asnaghi et al. [35] for elliptical foil. This study explored the mitigation of tip vortex cavitation inception by applying different roughness configurations. The results showed that the application of roughness on a small area of the foil section can prevent the performance degradation and a decrease in the inception of tip vortex cavitation can be achieved around $33 \%$ compared to the smooth condition.

There are limited studies in the literature investigating the impacts of the roughness on propellers in terms of noise and cavitation characteristics. One of the earliest studies was conducted by Van der Kooij [36] with a comparison between roughened and smooth propeller blades. In Van der Kooji's study [36], cavitation bubbles were generated by electrolysis currents varying from 0 to $2.4 \mathrm{~A}$ for the smooth blades. Additionally, the artificial roughness was used together with an electrolysis current of 2.4 A on the leading-edge part of the propeller blades for the roughened blades case. Furthermore, generated noise fluctuations through increasing cavitation bubble densities were investigated in 
model scale. Results showed that with the rising number of bubbles at the beginning, there is an increase in the noise; however, there is a remarkable drop when the numbers of bubbles become larger for both roughened and smooth cases until $63 \mathrm{~Hz}$. Krüger et al. [32] stated that Philipp and Ninnemann [37]'s study showed the application of roughness on a small area at the suction side of the blade close to the trailing edge could destabilise the tip vortex formation, hence its early breakdown. Cong et al. [38] conducted an experimental study and tested the cavitation characteristics of a composite coating on a propeller. Besides, they used the coating in full-scale ship propellers for more than a year. Their results presented that there was not a significant impact on thrust and torque characteristics of the propeller and the biofouling accumulation was able to be removed easily with high-pressure water. Korkut and Atlar [39] conducted an experimental study to investigate the differences between a coated and uncoated propeller in terms of hydrodynamic efficiency, cavitation, and noise characteristics at model scale. In their study, the first step was to compare the coated and uncoated propeller efficiencies in open water tests. The experiment results showed a good agreement with the uncertainty level of the open water tests, which was 3\%, similar to Mutton et al. [40]. After that, cavitation inception measurements were conducted for the coated and uncoated propeller blades in both uniform and non-uniform flow cases. The results, remarkably, indicated that there is no marked difference between coated and uncoated propellers cavitation characteristics. When noise levels were compared between coated and uncoated propeller, the results showed that while coating decreased the noise levels at higher advanced coefficients, at lower advance coefficients, noise levels were increased.

In addition to the studies mentioned above, there are also other studies related to cavitation and turbulence in the literature. Ji et al. [41] investigated the unsteady cavitation turbulent flow around the conventional marine propeller in a non-uniform wake. In the numerical calculations, $k-\omega$ SST turbulence model and sliding mesh approach were used to predict the excited pressure fluctuations. The results showed that acceleration caused by cavity volume changes is the main source of the pressure excitations induced by the propeller. Long et al. [42] studied verification and validation procedures for the URANS simulations of the turbulent cavitating flow around a Clark-Y hydrofoil. Density Corrected Model (DSM) was used to simulate the unsteady cavitation flow with the Zwart cavitation model. Different Richardson extrapolation-based uncertainty methods were explored for cavitating flow simulations. It was found that predicted velocity distributions, the transient cavitation patterns and the effects of the vortex stretching are strongly influenced by the grid resolution. Long et al. [43] simulated the cavitating flow around the highly-skewed propeller behind the hull condition using RANS and homogenous cavitation models. The relative vorticity transport equation was used to examine the influence of cavitation on the vorticity transport. The results showed that the relative 
vorticity distribution was affected by the leading-edge vortex, mass transfer and side re-entrant jet. Long et al. [44] simulated the cavitating flow around the Clark-Y hydrofoil using LES approach. This study was the first practical application of LES together with the Verification and Validation (V\&V) in cavitating flow. The time evolution process of the periodic cavity shedding was predicted with the numerical simulations and the results were compared with the experimental data. The requirement of the grid resolution was comprehensively discussed for V\&V study using LES method. In the study of Long et al. [45], cavitating flow both around the conventional marine propeller (CP) and a highly skewed marine propeller (HSP) was solved using LES. In the computations, LES V\&V was performed for two propellers with a simplified three equations method. In addition to this, the cavitation-vortex interactions were investigated using the relative vorticity transport equation. The crucial flow structures including the tip vortex, leading edge vortex, trailing vortex and internal jet were successfully simulated with the LES simulations.

\section{THEORETICAL BACKGROUND}

\subsection{Hydrodynamic Model}

The hydrodynamic simulations are performed by using the commercial computational fluid dynamics (CFD) solver; Star CCM+ [46] under non-cavitating and cavitating conditions using the unsteady RANS solver. The CFD method is based on the discretisation of the Navier-Stokes equations.

Cavitation is a phase change, and it occurs when the static pressure at a particular location within the liquid falls below the saturation vapour pressure in the forms of cavitation bubbles which consist of cavitation nuclei filled with either vapour, gas or a combination of them. The adopted solver (i.e. Star $\mathrm{CCM}+$ ) uses the homogeneous seed-based approach for cavitation and gas dissolution models. Moreover, it is assumed that seeds are spherical and uniformly distributed in the liquid, and all seeds initially have the same radius [46].

The unsteady RANS equations are solved for a homogeneous mixture with a phase change (i.e. liquid and vapour) to impose the cavitation. The physical properties of the mixture can be written as follows;

$$
\begin{aligned}
& \rho_{\text {mix }}=\alpha \rho_{\text {vapour }}+(1-\alpha) \rho_{\text {liquid }} \\
& \mu_{\text {mix }}=\alpha \mu_{\text {vapour }}+(1-\alpha) \mu_{\text {liquid }} \\
& u_{\text {mix }}=\alpha u_{\text {vapour }}+(1-\alpha) u_{\text {liquid }}
\end{aligned}
$$

where, $\rho$ is the density, $\mu$ is the dynamic viscosity, $\alpha$ is the gas volume fraction, and $u$ is the velocity. The final form of the continuity and momentum equations under incompressible assumption can be 
rewritten by introducing the volume fraction and additional source term on the right-hand side of the equations to explain the evaporation and condensation process as shown in following equations,

$$
\begin{aligned}
& \nabla \cdot\left(u_{\text {mix }}\right)=\left(\frac{1}{\rho_{\text {liquid }}}-\frac{1}{\rho_{\text {vapour }}}\right) \dot{m} \\
& \frac{\partial u_{\text {mix }}}{\partial t}+\nabla\left(u_{\text {mix }} u_{\text {mix }}\right)=-\frac{1}{\rho_{\text {mix }}} \nabla p+\nabla \cdot T_{R e}+F
\end{aligned}
$$

Here, $T_{R e}$ is the Reynolds Stress Tensor and it is calculated using the turbulence closure equations, $F$ is the additional momentum sources, and $\dot{m}$ is the mass flow rate per unit volume. In the solver, the Schnerr Sauer Cavitation Model, which is based on the reduced Rayleigh Plesset equation, is used with VOF (Volume of Fluid) approach to model the sheet and tip vortex cavitation. The cavitation model neglects the influence of the bubble growth \& collapse rates, viscous and surface tension effects [46]. In the solver, the cavities are identified using the vapour fraction (i.e. both liquid and water phases) and governed by same equations of motions. Using the $n_{0}$ (number of seeds per unit volume of liquid) and $R$ (local bubble radius), the mass transfer rate per unit volume $\dot{m}$, to solve Equation 4 and 5 , is obtained as follows:

$$
\dot{m}=\frac{\rho_{\text {liquid }} \rho_{\text {vapor }}}{\rho}(1-\alpha) \frac{3}{R} \sqrt{\frac{\frac{2}{3}\left(p-p_{\text {vapor }}\right)}{\rho_{\text {liquid }}}}
$$

\subsection{Hydroacoustic Model}

The potential or viscous based solvers are firstly applied for the determination of the hydrodynamic flow field around the propellers. Due to the higher computational cost of the Direct Numerical Simulations (DNS) and their inherent incapabilities for the sound propagation under the incompressibility assumption, the hybrid approach (i.e. hydrodynamic solver + acoustic analogy) are commonly employed for the prediction of the URN. In this approach, the source field is firstly identified using the incompressible solver. Later, the acoustic analogy is adopted for the noise propagation to the desired locations in the far-field. The most commonly used acoustic analogy is the Ffowcs Williams-Hawkings (FW-H) equation [47] which is the extended formulation of Lighthill's equation [48]. It is to be noted that the FW-H equation was initially developed for aeroacoustic calculations [49]. However, it has started to be used in maritime applications by taking the contribution of non-linear noise terms into account.

$\mathrm{FW}-\mathrm{H}$ equation is the rearranged form of the continuity and momentum equations into wave equations [47]. The different noise generation mechanisms are identified in the right-hand side of the equation using three various noise sources such as monopole (which is also called thickness), dipole 
(loading term), and quadrupole (non-linear) terms. The contribution of the monopole and dipole noise terms are evaluated by solving the surface integrals on the noise source. However, the quadrupole noise term needs volume integral solution. In the aero-acoustics field, the quadrupole noise term becomes important, especially at high supersonic or transonic regimes. Outside this specific domain, the flow field is generally dominated by linear noise terms (monopole and dipole) in most aeronautical problems which enables the evaluation of radiated noise by taking only two linear terms into account. Thus, this form of the FW-H equation is also known as Farassat $1 \mathrm{~A}$ solution [50]. However, due to the contribution of vorticity and turbulence (i.e. non-linear noise terms) on underwater pressure fields, especially in the far-field, the quadrupole noise sources need to be included in the calculations [51], [52]. Thus, instead of solving the integral volume terms which are computationally expensive and more sensitive to the accuracy of the input data, the porous or permeable formulation of $\mathrm{FW}-\mathrm{H}$ equation (P-FWH) becomes attractive since it includes all relevant non-linear noise sources induced in the propeller's slipstream [51]. The P-FWH equation was first implemented by Williams and Hawkings [47] and proposed as a possible solution of the FW-H equation by Di Francescantonio [53].

In the P-FWH approach, which was implemented in this study, two new variables are introduced as modified velocity $(U)$ and modified stress tensor $(L)$ as given in Equation 7 and 8. It should be noted that the monopole and dipole noise terms of Farassat $1 \mathrm{~A}[50]$ lose their original physical meaning. Thus, they become 'pseudo monopole' and 'pseudo-dipole' terms, and also include the contribution of the quadrupole (non-linear) term.

$$
\begin{aligned}
& U_{i}=u_{i}+\left(\frac{\rho}{\rho_{0}}-1\right)\left(u_{i}-v_{i}\right) \\
& L_{i}=P_{i j} \hat{n}_{j}+\rho u_{i}\left(u_{n}-v_{n}\right)
\end{aligned}
$$

Where $u_{i}$ and $v_{i}$ are the fluid and surface velocity components, respectively. $P_{i j}$ represents the compressive stress tensor $\left(\Delta P_{i j}=P_{i j}-p_{0} \delta_{i j}\right), n$ indicates the projection along the outward normal to the surface, and $\hat{n}$ is the unit vectors in the normal direction. For stationary integration domain and incompressibility assumption, the porous FW-H formulation can be written as [53];

$$
4 \pi p(x, t)=\int_{S} \frac{\rho_{0} \dot{U}_{n}}{r} d S+\int_{S} \frac{\dot{L}_{r}}{c_{0} r} d S+\int_{S} \frac{L_{r}}{r^{2}} d S+p_{Q}(x, t)
$$

where, $r$ is the distance between the noise source and the receiver, $c_{0}$ is the speed of sound, $p$ is the acoustic pressure, subscripts $r$ and $n$ define the dot product of a quantity with a unit vector either radiation or normal directions, respectively. The last term of Equation 9 represents the non-linear contribution of the noise sources outside of the integral surface in the fluid domain. It is considered 
that the extension of the integral surface encompasses all possible relevant non-linear noise sources; thus, the non-linear contribution outside of the porous domain can be neglected.

\subsection{Roughness Model}

The flow regime over a rough surface can be correlated with the interaction between the roughness elements and the boundary layer regions. If the roughness elements are small and hence, the linear sublayer contains these roughness elements, the flow regime is considered hydraulically smooth. In this case, fluid viscosity damps out the perturbations generated by the roughness elements, and hence there is no additional form drag due to roughness elements. If some part of roughness elements penetrates beyond the linear sublayer, they cause form drag and eddy shedding. The flow regime is called 'intermediate or transitional regime' where turbulent eddies generated by roughness elements, form drag on the elements and viscous drag affect the overall skin friction. If most of the roughness elements penetrate beyond the linear sublayer, the viscous sublayer vanishes, and form drag on the roughness elements becomes the dominant parameter on the momentum deficit. The flow regime is called fully rough in which skin friction becomes independent of Reynolds number, and viscous effects are no longer important [54], [55]. As shown in Figure 2, the given roughness conditions are assumed to imply that the flow is in the fully rough regime. Therefore, the velocity distribution in the log-law region can be described by Equation 10 .

$$
U^{+}=\frac{1}{\kappa} \ln \left(y^{+}\right)+B-\Delta U^{+}
$$

where, $\kappa$ is the von Karman constant, $y^{+}$is the non-dimensional normal distance from the boundary, $B$ is the smooth log law intercept. Roughness causes a downward shift in the velocity profile, which is commonly shown as $\Delta U^{+}$, as shown in [56]. The roughness function can be written as a function of the roughness Reynolds number as given in Equation 11.

$$
k^{+}=\frac{k U_{\tau}}{v}
$$

where $U_{\tau}$ is the friction velocity, $k$ is the roughness length, and $v$ is the kinematic viscosity. It is important to note that roughness function vanishes for the smooth case $\left(\Delta U^{+}=0\right)$, whereas it is determined experimentally for the rough cases. In this regard, Uzun et al. [25] conducted an extensive series of towing tank tests of flat plates which are covered by 3D printed barnacle tiles in varying sizes, coverages and settlement patterns. Test configurations include three different barnacle sizes, percentages of coverage area ranging from $10 \%$ to $50 \%$ and two different settlement patterns.

First, $\Delta U^{+}$, and corresponding $k^{+}$values, provided in Uzun et al. [25], were utilised in the wall function of the CFD software. After that, CFD simulations were performed based on the modified wall function 
of CFD model to predict the effect of roughness on the propeller blades. It is important to note that the roughness is homogenously distributed along with the propeller blades.

Table 1 tabulates the roughness length scales of the rough surfaces and Figure 2 illustrates the roughness functions along with the proposed roughness functions of Grigson [57], loselevich and Pilipenko [58] and Demirel et al. [6]. As seen in Figure 2, the roughness functions show excellent agreement with the Colebrook type roughness function of Grigson [57]. Further details on the roughness functions and the experiment can be found in Uzun et al. [25].

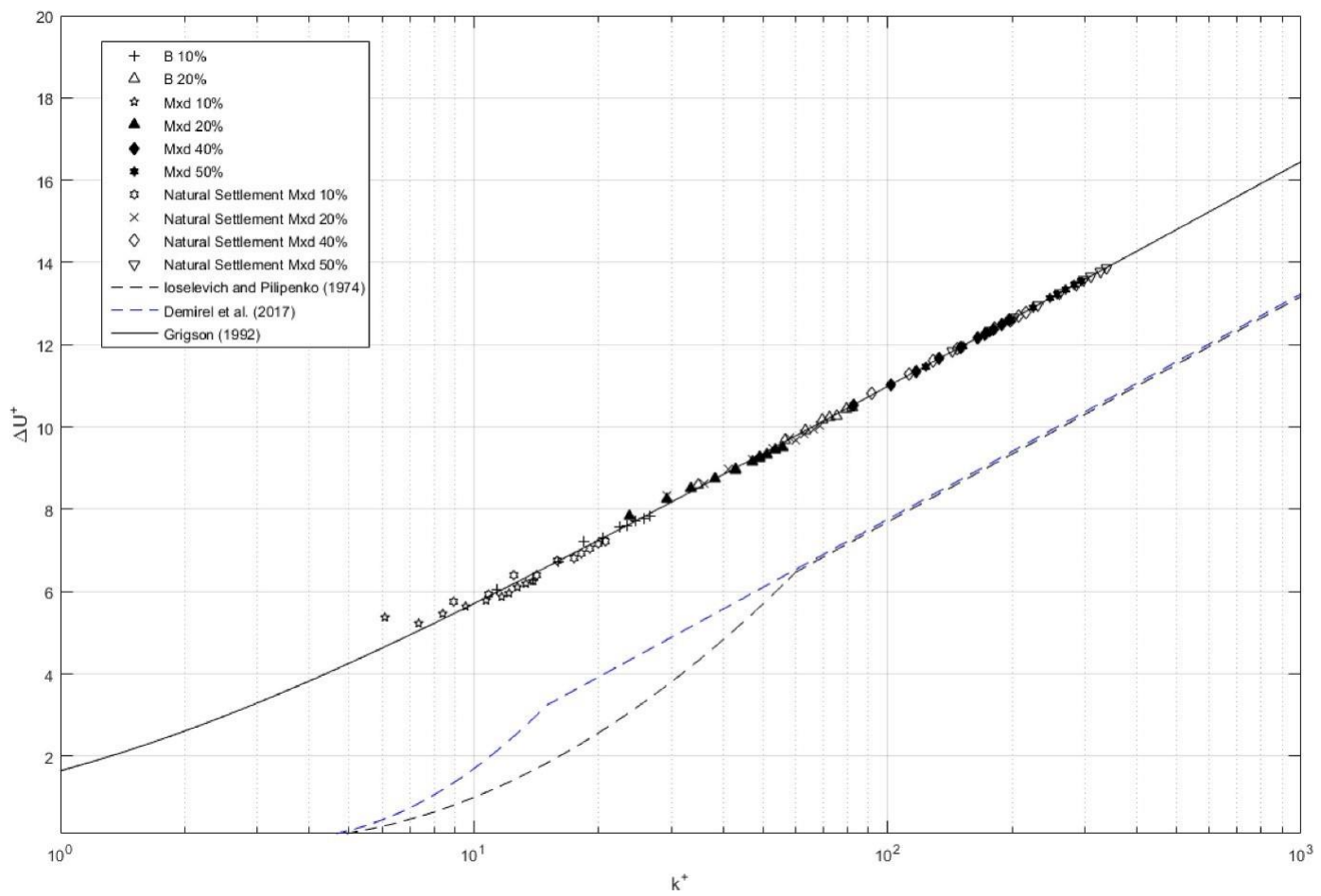

Figure 2. Roughness functions for various test surfaces, adapted from Uzun et al. [25] 
Table 1. Roughness length scales of test surfaces, adapted from Uzun et al. [25]

\begin{tabular}{cccc}
\hline Test Surfaces & $\begin{array}{c}\text { Surface } \\
\text { Coverage }(\%)\end{array}$ & $\begin{array}{c}\text { Barnacle } \\
\text { Height } h \\
(\mathrm{~mm})\end{array}$ & $\begin{array}{c}\text { Representative } \\
\text { roughness height } \\
k_{G}(\mu \mathrm{m})\end{array}$ \\
\hline Mix & 10 & $5,2.5,1.25$ & 94 \\
NS Mix & 10 & $5,2.5,1.25$ & 136 \\
Mix & 20 & $5,2.5,1.25$ & 337 \\
NS Mix & 20 & $5,2.5,1.25$ & 408 \\
\hline
\end{tabular}

It should be noted that resolving the flow around the roughness parameters, in which roughness elements need to be modelled physically in the computational domain, gives a more realistic solution, but it requires high computational power. The alternative approach is to model the flow around the roughness elements, which requires comparatively lower computational power. Although this approach simplifies the geometry of roughness elements and the physic of the flow is not precisely modelled, it is more effective in terms of applicability and computational source [35].

\section{NUMERICAL MODEL}

\subsection{Geometry and Test Cases}

The benchmark INSEAN E779A propeller geometry [59] was selected for the investigation of roughness on propeller hydrodynamic characteristics, cavitation, and hydroacoustic performance. The main particulars and general view of the four-bladed marine propeller can be seen in Table 2 and Figure 3, respectively.

Table 2. The main particulars of the propeller.

\begin{tabular}{lcl}
\hline Number of Blades & $Z$ & 4 \\
Diameter $(\mathrm{m})$ & $D$ & 0.227 \\
Pitch Ratio & $(P / D)$ & 1.1 \\
Expanded Area Ratio & $\left(A_{E} / A_{0}\right)$ & 0.69
\end{tabular}

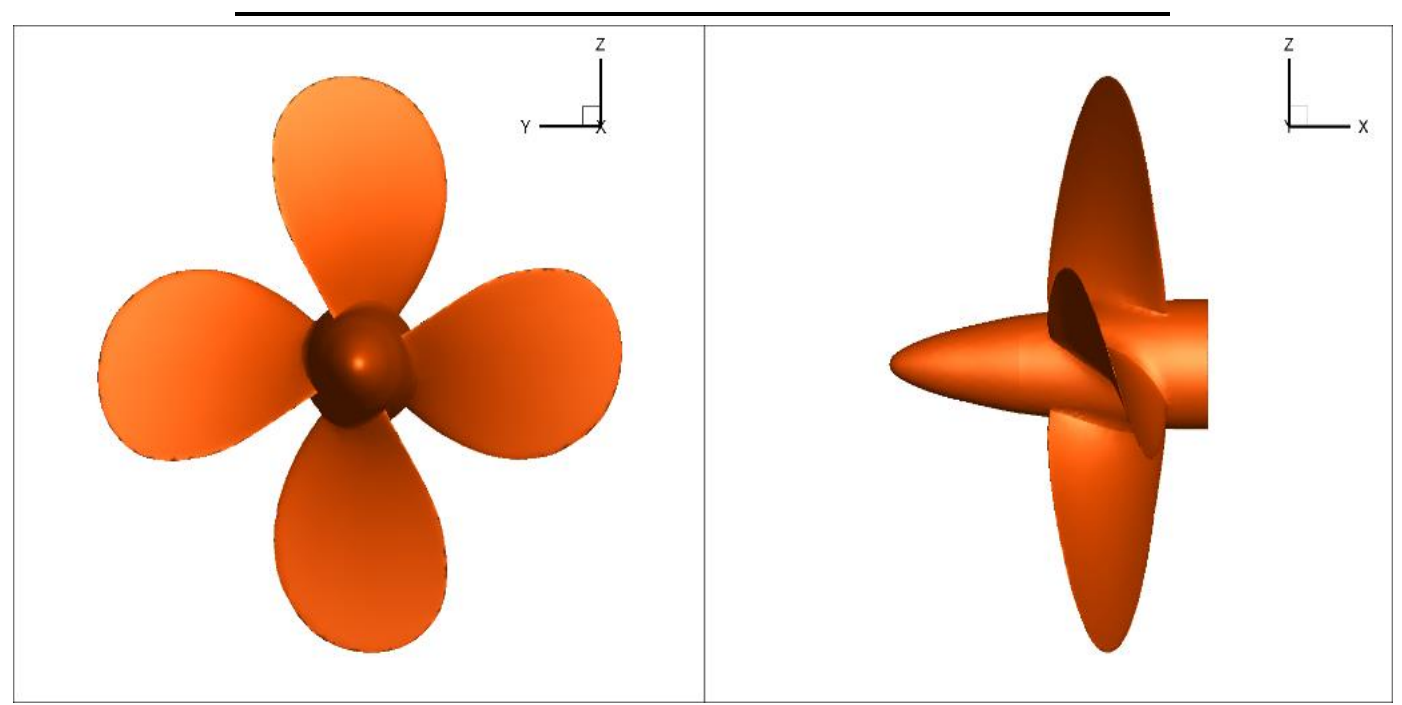

Figure 3. Back and side views of the INSEAN E779A propeller 
Table 3 tabulates the selected tests cases for the investigation of the roughness on the open-water (OW) hydrodynamic performance, cavitation and URN characteristics. In non-cavitating OW simulations, the propeller rotational rate $(n)$ was kept constant around $12 \mathrm{rps}$ in correspondence to the experiment and inflow velocity $\left(V_{A}\right)$ was set according to the advance ratio $(J)$. In cavitation simulations, the propeller rotational rate was set to $36 \mathrm{rps}$ as in experimental conditions, and vapour pressure was kept constant $\left(P_{V}\right)$ as $2337 \mathrm{~Pa}$. The hydroacoustic performance of the propeller was predicted under cavitating conditions. The four different roughness configurations (M10, M20, NSM10 and NSM20) were implemented for each case under non-cavitating and cavitating conditions as given in Table 3.

Table 3. Description of the test matrix

\begin{tabular}{lccc}
\hline & Non-cavitating cases & \multicolumn{2}{c}{ Cavitation and URN cases } \\
\hline Condition & $J$ & $J$ & $\sigma$ \\
Smooth & & & \\
Rough & $0.397 ; 0.498 ; 0.596 ; 0.795$ & 0.71 & 1.763 \\
\hline
\end{tabular}

\subsection{Computational Domain and Boundary Conditions}

A cylindrical computational domain was utilised for the numerical simulations. The domain length was set as 3D and 7D from the upstream and downstream of the propeller centre, respectively. The radial extension was set as 8D. The computational domain was divided into three parts as a static, rotating and noise region, as shown in Figure 4. The transition between the regions was provided by sliding interfaces. The wall with a no-slip condition was imposed on the propeller blades and hub to satisfy the kinematic boundary condition. The positive $X$ direction was defined as velocity inlet, whereas the pressure outlet was defined in the negative $X$ direction. The remaining surfaces of the computational domain were identified as symmetry planes. The detailed information about the boundary conditions is given in Table 4. It is to be noted that the same computational domain was used for non-cavitating and cavitating simulations. 


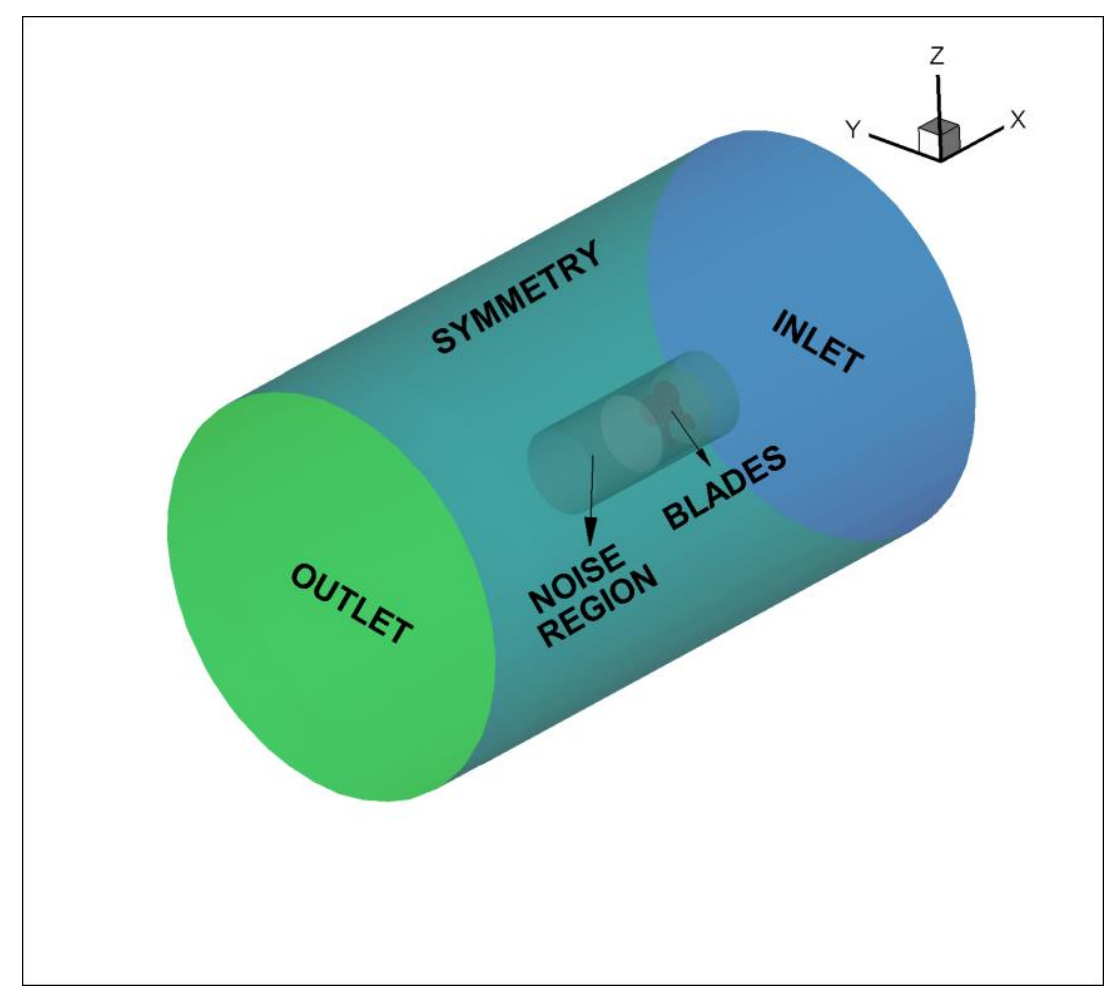

Figure 4. Representation of the computational domain

Table 4. Boundary Conditions

\begin{tabular}{cc}
\hline Boundary Conditions & Type \\
\hline Inlet & Velocity Inlet \\
Outlet & Pressure outlet \\
Sides of the domain & Symmetry \\
Propeller blades and shaft & Wall \\
\hline
\end{tabular}

\subsection{Grid Structure and Adaptive Mesh Refinement (AMR) Technique}

The adaptation of the suitable grid structure can be considered as one of the challenges in most of the CFD applications, which is closely linked to discretisation errors in the numerical solvers. Thus, it needs to be appropriately defined to increase the accuracy of the solution. As it is known, the flow around the propeller blades is a complex phenomenon and sensitivity of the grid structure can considerably affect the numerical results. Due to the further complexity of the cavitation phenomenon and its dynamics, the interaction between the turbulent and cavitating flow becomes even more complicated compared to the interaction with non-cavitating flow. Thus, once the grid is sufficiently enough on the propeller blades, it can be considered that the grid structure for the prediction of global performance characteristics (i.e. thrust and torque) is less sensitive than the cavitation and URN simulations [60]. Besides, one of the challenges of these simulations is to model the tip vortex cavitation (TVC) (i.e. prediction of minimum pressure in the vortex cores), which is directly related to the numerical method, grid size \& grid properties in the vortex core regions and boundary layer resolution. Thus, in 
this study, two different mesh algorithms were used for the non-cavitating and cavitating flows and associated URN simulations.

It is a well-known fact that the use of fine grid application in the entire propeller slipstream region increases the total cell number and computational time. This enforces the simulations become unfeasible for most of the practical applications. In order to bring a practical solution to this problem, recently, various special meshing techniques, such as Adaptive Mesh Refinement (AMR), are locally employed in the propeller's slipstream to resolve the TVC better as reviewed in the following.

The AMR mesh technique is a dynamic method that refines or coarsens cells in the specified regions of the computational domain based on the adaptive mesh criteria. In this technique, the solution quantities are automatically interpolated to the new adapted mesh. The application of the AMR technique is not easy because of the difficulties in the determination of refinement criterion, which can be generally selected as either pressure or vorticity. As a pilot study, the first application of the AMR technique was conducted using the pressure as the refinement criterion in our test cases. However, as the pressure field looks similar to Gaussian function in the transversal direction of the tip vortex, which is clearly stated in the study of Yvin and Muller [61], it is hard to determine whether the minimum pressure is always located in the center of the tip vortex or not. As stated in the study of Yvin and Muller [61], a refinement criterion should not be based on the pressure itself, and hence other criteria, e.g. vorticity-based ( $Q$-Criterion), can be useful. Besides, the total element count can be lower using the vorticity-based refinement criterion when compared to the pressure-based. Therefore, the vorticity was selected as a refinement criterion in this study to observe the TVC.

Additionally, by considering the cell size (or bubble radius) in the tip vortex region, it is vital to define the bubble radius sufficiently for the observation of TVC. Within this framework, Asnaghi [62] and Asnaghi et al. [63] conducted a comprehensive numerical study for accurate solution of tip vortex for foil section using different cell numbers in the vortex cores. Also, the series of cavitation tests using different model scale propellers were conducted by Kuiper [64] for the investigation of the bubble, sheet and tip vortex cavitations. Kuiper [64] investigated the relationship between cavitation index $(\sigma)$ and core radius $\left(a_{c}\right)$. According to experimental results, the core radius at the inception was measured around $0.25 \mathrm{~mm}$. Therefore, by taking the crucial suggestions of the studies (e.g. [62], [63], [64]) as well as the authors experience, the bubble radius (or mesh size) in the vortex core was kept as $0.2 \mathrm{~mm}$ in this study. The different forms of the AMR technique were implemented in the literature for TVC observations in the propeller's slipstream, either modelling only one blade (e.g. [65]) or all blades (e.g. [66]) by using different refinement criterion.

The proposed AMR technique composed of two main steps; namely $1^{\text {st }}$ and $2^{\text {nd }} A M R$, to make the simulation more computationally affordable. At the $1^{\text {st }}$ step, once the flow field converges using the 
initial mesh (i.e. without AMR), the cavitating tip vortex areas can be visualised using the threshold value of vorticity-based $Q$-criterion. When the field of interest for the TVC was determined, the $1^{\text {st }}$ AMR was adopted using the field function refinement table in all directions $\left(Q_{C 1}=190.0001 /\right.$ $s^{2}$ in our case). The refinement table utilises the vorticity magnitude as an AMR refinement criterion. In this way, the desired cells were created in the specified areas where the magnitude of vorticity is higher than the threshold value (i.e. user-specified value). At the $1^{\text {st }}$ stage, the trajectory of TVC was determined using a relatively coarse grid size in the vortex cores. After that, the $2^{\text {nd }}$ stage of AMR was implemented to observe the final extension of TVC $\left(Q_{C 2}=2.500 .0001 / \mathrm{s}^{2}\right.$ in our case $)$.

From the hydroacoustic point of view, the requirements of the suitable grid structure were discussed in the $27^{\text {th }}$ ITTC discussion forms [67]. On the contrary to traditional hydrodynamic simulations, the application of high-quality mesh was suggested for accurate prediction of the URN. Besides, the ITTC recommended more studies to be conducted in this research field for verification and validation.

In the numerical calculations, two main criteria should be met by the adopted grid structure for hydroacoustic simulations [46]:

- $\quad$ The grid should be sufficient to resolve or model the sound generating turbulence scales as well as cavitation dynamics.

- It should be able to resolve the propagation of acoustic waves towards the locations of the desired distance in the far-field.

Furthermore, the numerical hydroacoustic solvers suffer from numerical noise which is mainly attributed to sliding interfaces or mesh transitions in the noise region. Thus, any mesh change inside the noise region was prevented to avoid the numerical noise or spurious noise as much as possible during the simulation [46]. The unstructured grid was used to discretise the computational domain by using trimmer mesh approach. An automated mesh, which is coupled with the AMR technique, was adopted to consider both hydrodynamic and hydroacoustic perspective. Figure 5 shows the grid structure implemented at the $1^{\text {st }}$ and $2^{\text {nd }}$ stages of AMR in addition to the general view of the grid structure around the propeller blades. 

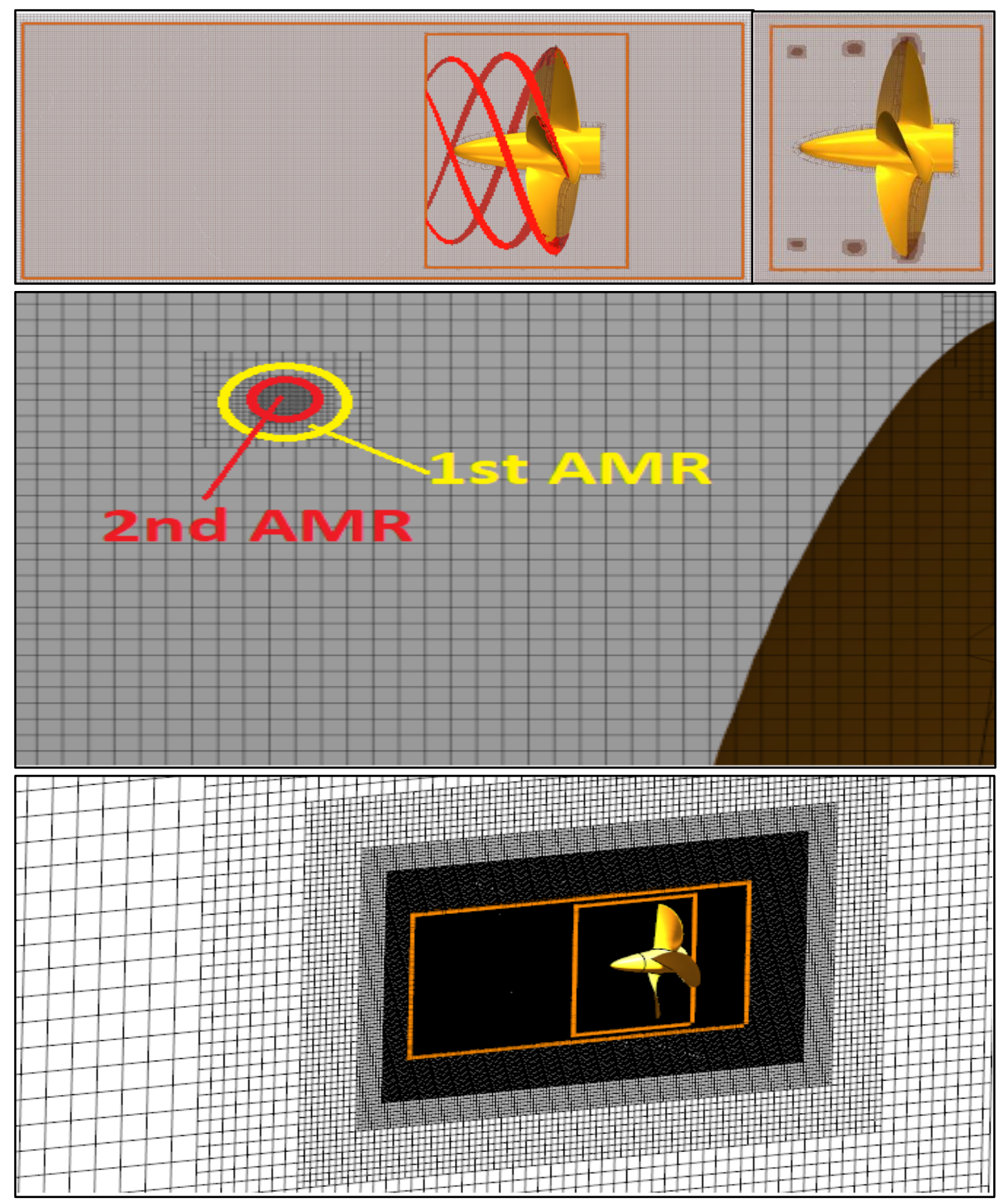

Figure 5. Visualisation of $1^{\text {st }}$ and $2^{\text {nd }} A M R$ regions and general mesh view 
Table 5 indicates the total element counts with and without AMR for cavitation and noise simulations. As it can be seen clearly, in the presence of AMR, the total element count increased around $5 \mathrm{M}$ by modelling all propeller blades. If the $2^{\text {nd }}$ stage of AMR were directly implemented for the simulation with smaller grid sizes, the total element count would be around $36 \mathrm{M}$, which would not be practical for CFD applications in engineering.

Table 5. Calculated cell properties and total element count

\begin{tabular}{cccc}
\hline & $\begin{array}{c}\text { Surface size in the } \\
\text { vortex core }(\mathrm{mm})\end{array}$ & $\begin{array}{c}\text { Refinement } \\
\text { Factor }\end{array}$ & $\begin{array}{c}\text { Number of Cells } \\
\text { (Million) }\end{array}$ \\
\hline Without AMR & $0.007 \mathrm{D}$ & - & 12.188 .667 \\
$1^{\text {st }}$ AMR & $0.00175 \mathrm{D}$ & Cell Size/4 & 16.001 .134 \\
$2^{\text {nd }}$ AMR & $0.000875 \mathrm{D}$ & Cell Size/8 & 16.942 .008 \\
\hline
\end{tabular}

It should be noted that the threshold value of the vorticity-based $Q$ criterion depends on the operating condition of the propeller, and it needs to be set for each condition before creating cells using the refinement table. Additionally, the procedure for the observation of TVC can be repeated 3 or 4 times. However, from our experience and some internal test studies, implementing the above procedure more than two times does not give any benefit in terms of the extension of TVC in model scale. In contrast, the total element count increases as the new specified vorticity region (i.e. tip vortex region) extends beyond the region which is created in the $2^{\text {nd }}$ stage of the AMR. Therefore, it is suggested to apply the procedure two times.

\subsection{Solution Algorithm}

In the numerical calculations, the RANS method with k- $\omega$ SST turbulence model was used for both hydrodynamic and hydroacoustic simulations using all $y^{+}$wall treatment methodology. The secondorder scheme was used for the convection term and temporal discretisation. The segregated flow model was used with SIMPLE type algorithm between continuity and momentum equations.

Two different models were used for modelling the propeller rotational motion, namely Moving Reference Frame (MRF) and Rigid Body Motion (RBM or sliding interface). In the MRF approach, the governing equations are written in the rotating coordinate system of the propeller. It uses absolute velocities for the numerical solution. On the other hand, RBM refers to rotation of the computational domain with the same propeller rotational rate; thus, the governing equations are integrated in real times [68]. The MRF approach is generally preferred for the investigation of non-cavitating hydrodynamic performance of the propellers. In contrast, RBM is used to obtain the unsteady features of the flow field, cavitation, and hydroacoustic simulations. In this study, MRF and RBM were utilised for hydrodynamic and hydroacoustic simulations, respectively. 
The non-cavitating hydrodynamic simulations were conducted in a steady manner to obtain the global performance characteristics (i.e. thrust, torque and efficiency). In contrast, unsteady simulations were performed for the investigation of cavitation and hydroacoustic performance using 1 degree of propeller rotational rate (i.e. around $7.7^{*} 10^{-5}$ ). The simulations were performed using 40 -cores of the super computer system (ARCHIE-WeSt). The total solver elapsed time and CPU time is $\mathbf{5 7 . 7 7}$ hours and 2253.42 hours, respectively for each cavitating and noise simulations.

In order to include the effects of roughness on the numerical calculations, the selected $y^{+}$value needs to be higher than 30 and higher than $k^{+}$values recommended by Star CCM+ [46]. In other words, the distance of the first grid node to the wall must exceed the roughness height; otherwise, the roughness effect cannot be imposed on the numerical calculations.

The Schnerr-Sauer cavitation model was employed with the VOF approach to model the phase change in the cavitation simulations. The customisable cavitation parameters such as seed diameter and density are available in the facilities of Star $\mathrm{CCM}+[46]$. The number of nuclei might be significant for cavitation dynamics, and it is desired to have as same as with the experiment. However, it may not be possible to obtain properly from the experimental studies. Therefore, different commercial CFD solvers have their default values. The effects of the number of seed diameter and density were investigated comprehensively using Star CCM+ by Gaggero et al. [69] who found some minor difference in the TVC formation, but the consistency of the default values was presented. Thus, in this study, the default values provided by the commercial solver were selected as $10^{-6}$ and $10^{12}$ for seed diameter and density, respectively. The flow chart in Figure 6 summarises the numerical steps, which was applied in this study.

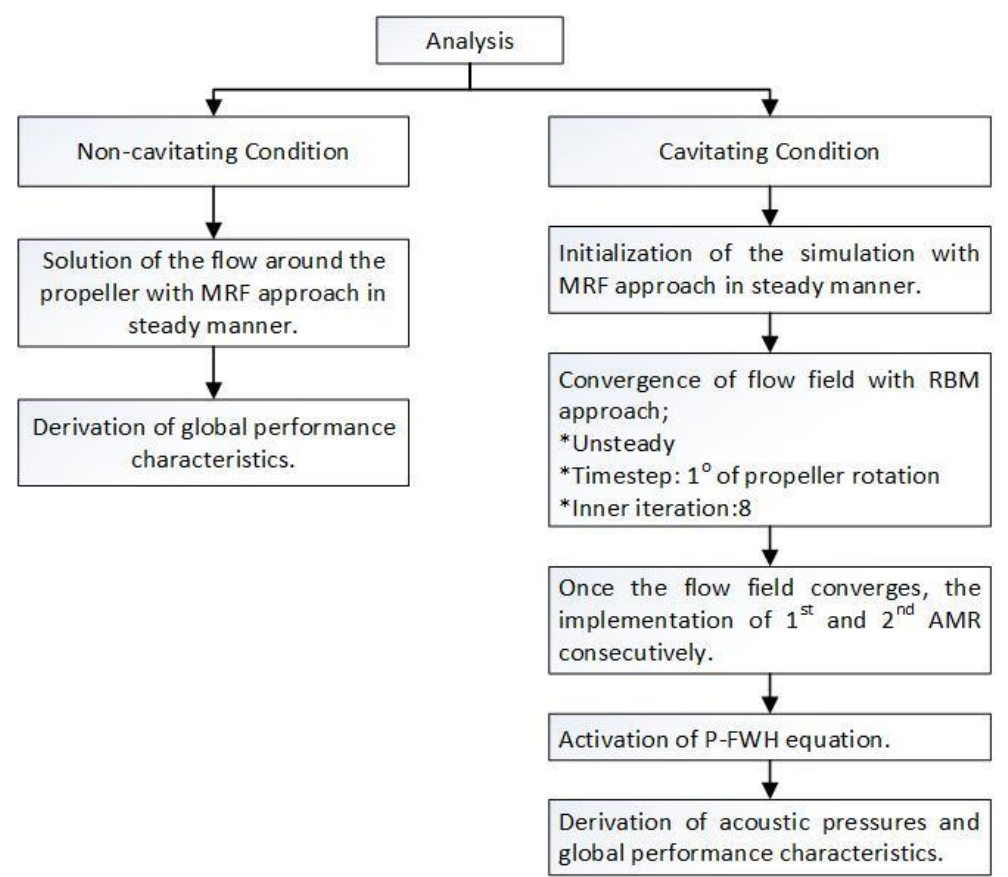

Figure 6. Flow chart for the numerical calculations 


\section{NUMERICAL RESULTS}

\subsection{Uncertainty Study}

The GCI (Grid Convergence Index) technique, which is suggested for the CFD validation studies in ITTC procedure [70], was employed to determine the uncertainty level of the numerical solution and sufficient grid spacing for the simulations. The $\mathrm{GCl}$ method based on Richardson extrapolation [71] was first proposed by Roache [72] and has been implemented in several studies in the literature.

In this method, the refinement factor $\left(r_{f}\right)$ was selected as $2^{1 / 2}$ which is generally employed in CFD applications. Three different solutions are desired to make an assessment of the uncertainty of the solution due to the spatial discretisation [72]. Therefore, three solutions were used for both hydrodynamic and cavitation + hydroacoustic simulations. The difference between solutions scalars $(\varepsilon)$ can be computed as in following;

$$
\varepsilon_{21}=\varphi_{2}-\varphi_{1}, \quad \varepsilon_{32}=\varphi_{3}-\varphi_{2}
$$

Here, $\varphi_{1}, \varphi_{2}, \varphi_{3}$ indicate the fine, medium, and coarse mesh grid solution, respectively. In this study, the solution scalars were selected as the thrust and torque coefficients. The convergence condition $(R)$ of the numerical solution can be checked using the following formulation and assessed for the range of $R$ values for the determination of the solution type [73];

$$
R=\frac{\varepsilon_{21}}{\varepsilon_{32}}
$$

- Oscillatory convergence: $-1<R<0$

- monotonic convergence: $0<R<1$

- oscillatory divergence: $R<-1$

- monotonic divergence: $R>1$

The extrapolated value can be calculated using the following equation;

$$
\varphi_{\text {ext }}^{21}=\left(r_{f}^{p o} \varphi_{1}-\varphi_{2}\right) /\left(r_{f}^{p o}-1\right)
$$

The approximate and extrapolated relative errors are also calculated as in following;

$$
e_{a}^{21}=\left|\frac{\varphi_{1}-\varphi_{2}}{\varphi_{1}}\right| \quad e_{\text {ext }}{ }^{21}=\frac{\left|\varphi_{\text {ext }}{ }^{12}-\varphi_{1}\right|}{\varphi_{\text {ext }}{ }^{12}}
$$


Finally, the uncertainty level of the numerical solution can be calculated by;

$$
\operatorname{GCI}_{\text {fine }}{ }^{21}=\frac{1.25 e_{a}^{21}}{r_{f 21}{ }^{p o}-1}
$$

where $p o$ is the apparent order of the method. The detailed procedure of the applied method can be found in the study of Celik et al. [74].

Table 6 shows the total element counts and solution scalars for the uncertainty study of non-cavitating hydrodynamic simulations. Here, $N_{1}, N_{2}$ and $N_{3}$ refer to element counts of fine, medium and coarse meshes, respectively. As a result of the uncertainty study, fine mesh was selected for the rest of the analysis under non-cavitating conditions both in smooth and rough cases.

Table 6. Spatial converge study for non-cavitating hydrodynamic simulations at $J=0.397$

\begin{tabular}{ccc}
\hline & $K_{T}$ & $10 K_{Q}$ \\
\hline$N_{1}$ & \multicolumn{2}{c}{6.725 .353} \\
$N_{2}$ & \multicolumn{2}{c}{2.962 .544} \\
$N_{3}$ & \multicolumn{2}{c}{1.121 .426} \\
$\varphi_{1}$ & 0.380 & 0.646 \\
$\varphi_{2}$ & 0.376 & 0.645 \\
$\varphi_{3}$ & 0.369 & 0.637 \\
$r_{\mathrm{f} 21}$ & \multicolumn{2}{c}{1.314} \\
$r_{\mathrm{f} 32}$ & \multicolumn{2}{c}{1.382} \\
$\varepsilon_{21}$ & -0.004 & -0.001 \\
$\varepsilon_{32}$ & -0.007 & -0.008 \\
$p o$ & 1.613 & 7.518 \\
$q$ & -0.196 & -0.196 \\
$s$ & 1 & 1 \\
$e_{a}{ }^{21}$ & 0.010 & 0.0015 \\
$e_{\text {ext }}{ }^{21}$ & 0.019 & 0.0002 \\
$\varphi_{\text {ext }}{ }^{21}$ & 0.387 & 0.646 \\
$R$ & 0.517 & 0.125 \\
$G C I_{\text {FINE }}(\%)$ & 2.376 & 0.028 \\
\hline
\end{tabular}

Table 7 tabulates the total element counts and solutions of different grids for the uncertainty study of cavitation and hydroacoustic simulations. It should be noted that the uncertainty study of cavitating and hydroacoustic simulations was conducted using initial mesh (i.e. without AMR) to decrease the computational cost. This is because the TVC does not affect the propeller global performance characteristics in the numerical calculations as in experiments. Similarly, a lack of effect of TVC on thrust and torque coefficients was found in the studies of Krasilnikov [75] and Gaggero et al. [69]. Consequently, the fine grid structure was selected only for cavitation and hydroacoustic simulations both in smooth and rough conditions. 
Table 7. Spatial Converge study for cavitating and hydroacoustic simulations

\begin{tabular}{ccc}
\hline & $K_{T}$ & $10 K_{Q}$ \\
\hline$N_{1}$ & \multicolumn{2}{c}{12.188 .667} \\
$N_{2}$ & \multicolumn{2}{c}{4.641 .416} \\
$N_{3}$ & \multicolumn{2}{c}{1.842 .196} \\
$\varphi_{1}$ & 0.2380 & 0.4340 \\
$\varphi_{2}$ & 0.2384 & 0.4353 \\
$\varphi_{3}$ & 0.2389 & 0.4616 \\
$r_{\mathrm{f} 21}$ & \multicolumn{2}{c}{1.379} \\
$r_{\mathrm{f} 32}$ & \multicolumn{2}{c}{1.360} \\
$\varepsilon_{21}$ & 0.0004 & 0.0012 \\
$\varepsilon_{32}$ & 0.0005 & 0.0263 \\
$p o$ & 0.817 & 9.600 \\
$q$ & 0.051 & 0.051 \\
$s$ & 1 & 1 \\
$e_{a}{ }^{21}$ & 0.0017 & 0.0028 \\
$e_{\text {ext }}{ }^{21}$ & 0.0056 & 0.0001 \\
$\varphi_{\text {ext }}{ }^{21}$ & 0.237 & 0.434 \\
$R$ & 0.80 & 0.05 \\
$G C I_{\text {FINE }}(\%)$ & 0.698 & 0.016 \\
\hline
\end{tabular}

\subsection{Hydrodynamic Results}

\subsubsection{Non-cavitating Condition}

\subsubsection{Smooth Case}

Figure 7 shows the validation of numerical open water test results with the experimental data for several advance coefficients in smooth condition. In this figure the propeller advance coefficient $(J)$, thrust coefficient $\left(K_{T}\right)$, torque coefficient $\left(K_{Q}\right)$ and open water efficiency $\left(\eta_{0}\right)$ are defined as;

$$
J=\frac{V_{A}}{n D}, K_{T}=\frac{T}{\rho n^{2} D^{4}}, K_{Q}=\frac{Q}{\rho n^{2} D^{5}}, \eta_{0}=\frac{J}{2 \pi} \frac{K_{T}}{K_{Q}}
$$

where $V_{A}$ is propeller advance speed (this is ground speed or incoming flow at infinity upstream) $(\mathrm{m} / \mathrm{s}), n$ is the propeller rotational rate (rps), $T$ is thrust $(\mathrm{N}), Q$ is propeller torque $(\mathrm{Nm}), \rho$ is the density of water $\left(\mathrm{kg} / \mathrm{m}^{3}\right)$. The Reynolds number of the propeller at $0.7 \mathrm{R}$ section is defined as;

$$
R e=\frac{c_{0.7 R} \sqrt{V_{A}^{2}+(0.7 \pi n D)^{2}}}{v}
$$

Here, $c_{0.7 R}$ is the chord length at $0.7 \mathrm{R}(\mathrm{m})$ and $v$ is the kinematic viscosity of water $\left(\mathrm{m}^{2} / \mathrm{s}\right)$. In the numerical calculations, Reynolds numbers $\left(R e_{0.7 R}\right)$ ranged from $2.5 \times 10^{5}-3.5 \times 10^{5}$. 
Figure 7 suggests that there is a satisfactory agreement between numerical and experimental results in spite of the underestimation of $K_{T}$ and hence $\eta_{0}$ values at higher $J$. The absolute differences between CFD and experiment are also given in Table 8.

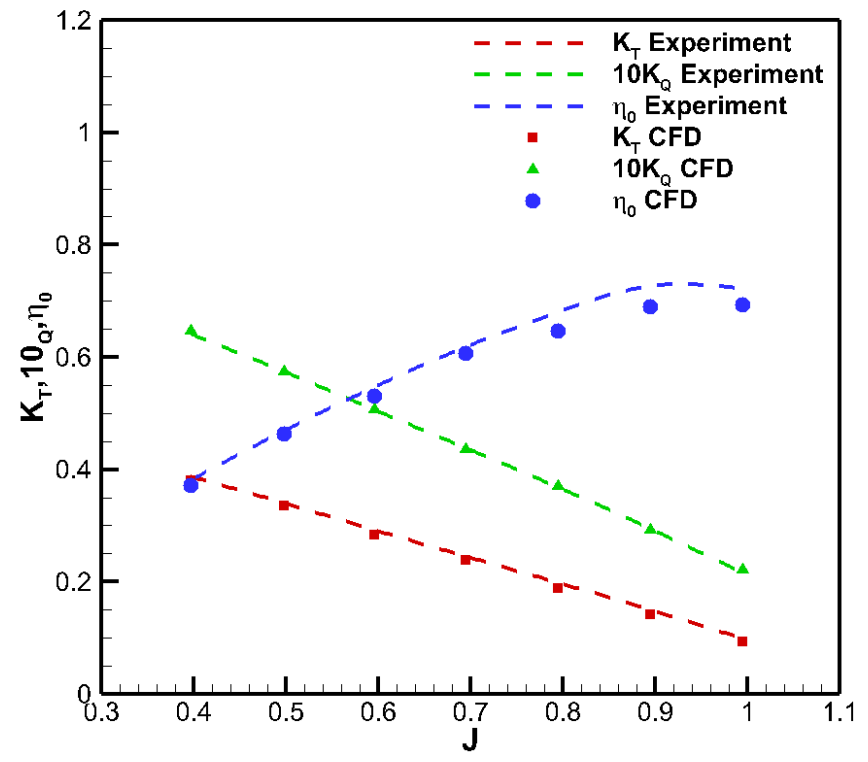

Figure 7. Validation of global performance characteristics in smooth condition

Table 8. The absolute differences between CFD and experiment for thrust, torque and efficiency

\begin{tabular}{cccc}
\multicolumn{4}{c}{ values. } \\
\hline$J$ & $K_{T}(\%)$ & $10 K_{O}(\%)$ & $\eta_{0}(\%)$ \\
\hline 0.397 & 1.809 & 0.724 & 2.514 \\
0.498 & 1.722 & 0.025 & 1.697 \\
0.596 & 1.670 & 0.073 & 1.598 \\
0.695 & 2.388 & 0.429 & 1.967 \\
0.795 & 3.885 & 0.860 & 3.051 \\
0.895 & 5.920 & 0.913 & 5.053 \\
0.995 & 5.980 & 2.232 & 3.833 \\
\hline
\end{tabular}

\subsubsection{Rough Case}

Figure 8 and 9 show the change in the propeller global performance characteristics under different roughness conditions, which are given in Table 1 . In the figures, zero roughness height indicates a hydraulically smooth condition. As it is expected, the presence of the roughness, depending on its height, will influence the boundary layer and hence will have significant impact on the hydrodynamic characteristics of propeller blade sections, including the drag and lift. Propeller roughness leads to an increase in the drag coefficient of the blades sections while reducing the lift coefficient. The reason behind the decreasing of the lift is the reduction in the circulation distribution around propeller blades due to roughness which consequently results in a reduction of thrust coefficient, as shown in Figure 8. The decrease in the $K_{T}$ values were predicted between around $5 \%$ and $18 \%$ at different advance 
coefficients due to the roughness. It is to be noted that the percentage decrease in the thrust coefficient remained almost similar (i.e. between $5 \%$ and $11 \%$ ) at first three advance coefficients. In contrast, the maximum decrease was observed at the highest advance ratio due to the relatively smaller $K_{T}$ value in smooth condition.

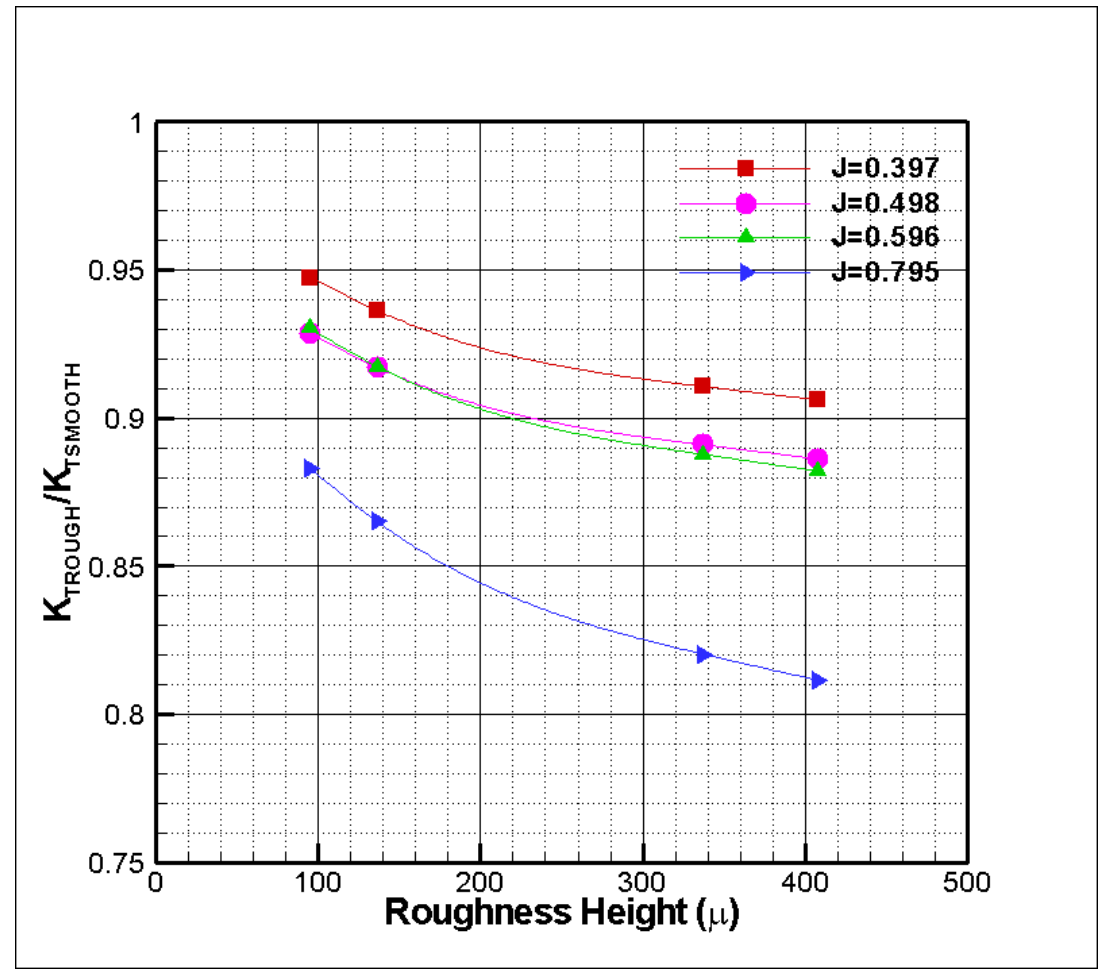

Figure 8. Change in $K_{T}$ values with different roughness conditions

Figure 9 indicates the change of the torque coefficient with different roughness heights. It should be borne in mind that the prediction of the torque strongly depends on the accurate estimation of the viscous forces in the boundary layer [76]. Since the roughness directly contributes to the shear and viscous forces in the linear sublayer, it causes an increase in torque. Additionally, the increase of the drag in the propeller section increases the torque, whereas it reduces the thrust. The increase in the $K_{Q}$ values were predicted between around $4 \%$ and $18 \%$ at different advance coefficients with an increase in roughness height. Similarly, the increase of torque changes approximately from $4 \%$ to $12 \%$ at $J=0.397,0.498$, and 0.596 with different roughness heights, while the maximum increase was computed at $J=0.795$ and more severe roughness condition (i.e. around $18 \%$ ). 


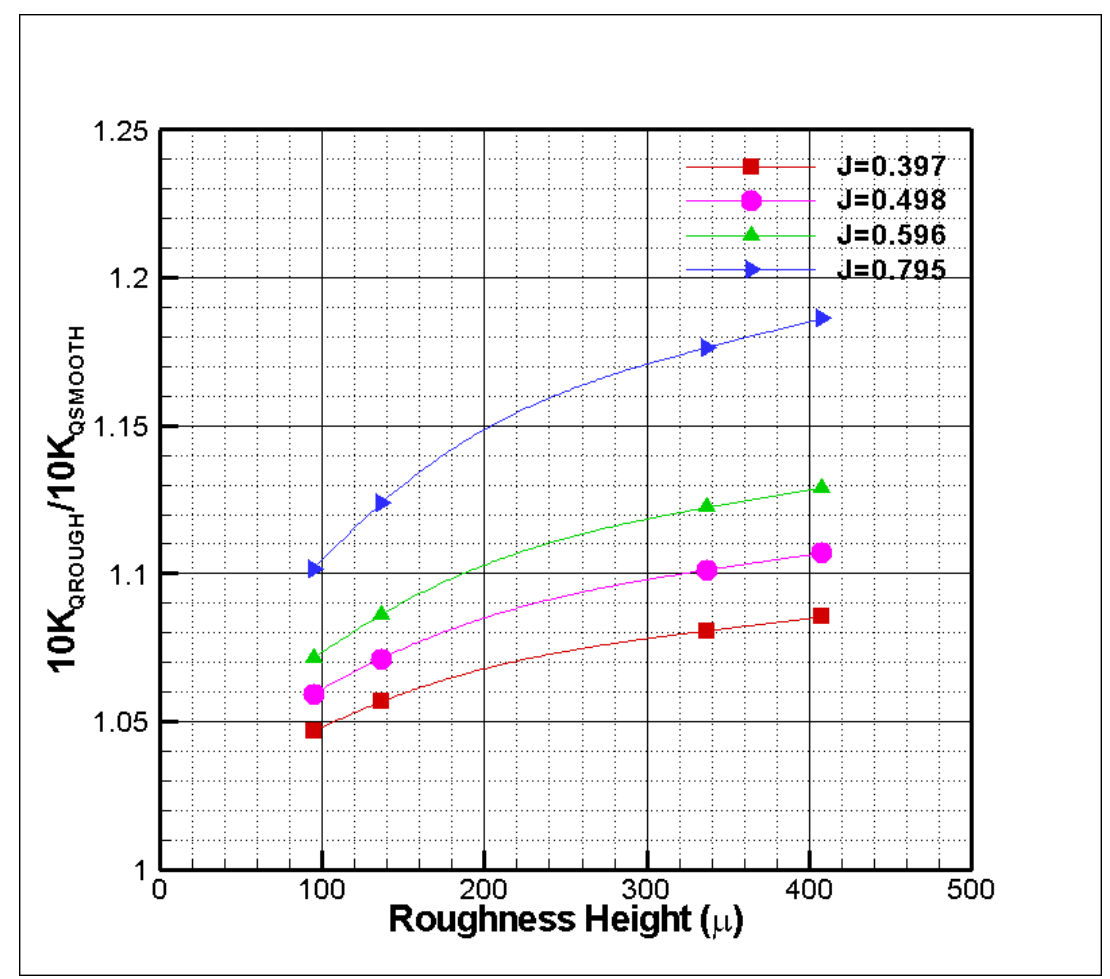

Figure 9. Change in $10 K_{Q}$ values with different roughness conditions

Without a doubt, an increase in torque and a decrease in thrust values cause a detrimental impact on propeller efficiency. The loss of propeller efficiency due to roughness can be seen in Figure 10 for different advance ratios and rough conditions. Depending on the roughness severity, the roughness increases the efficiency loss due to the increased viscous friction effect. Therefore, it is crucial to keep the propeller blades as smooth as possible from the efficiency point of view [77], [78].

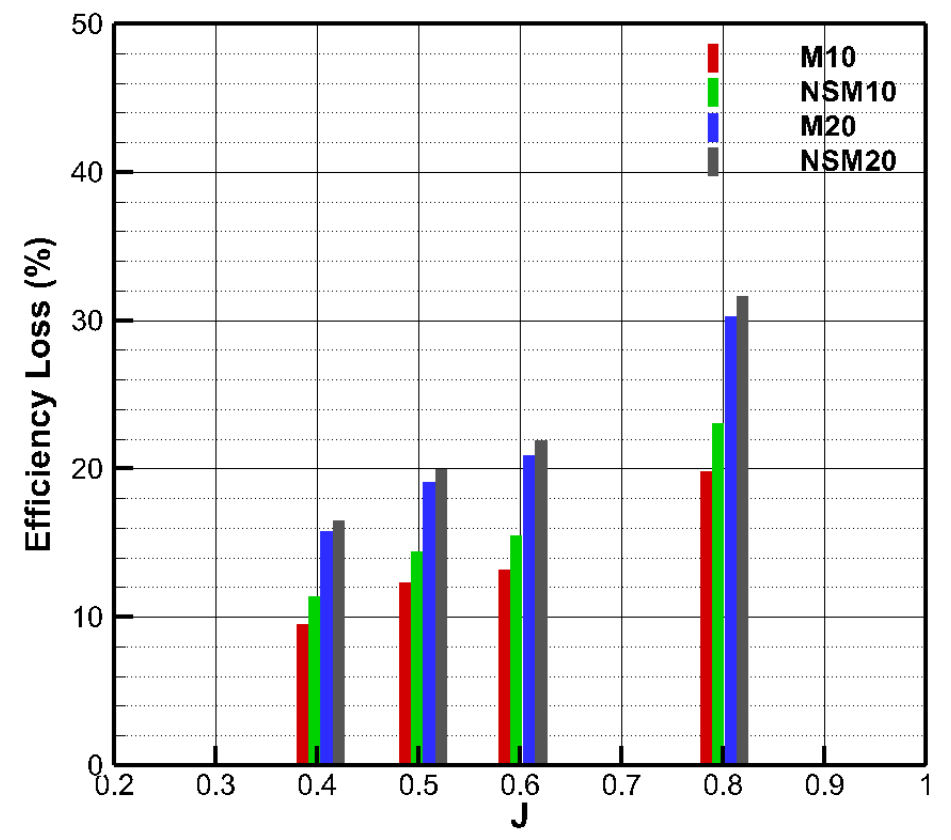

Figure 10. Efficiency loss due to roughness at different advance coefficients 
The scaling laws of the roughness strongly depend on the wall shear stress velocities at the propeller blade tips. The differences in wall shear stress velocities between the model scale and full scale cause significantly extended roughness height in model scale [32]. In order to replicate the same roughness impact on the model scale, the roughness height must be nearly the same order with the full-scale propeller. Since the geometric and hydrodynamic roughness similarity cannot be achieved together between the model and full-scale propeller, as stated in the study of Kruger et al. [32], the full-scale propeller efficiency is expected to be less influenced by the viscous effects than for the model scale propeller.

The significant loss can also be expected in propulsive efficiency due to the decrease in propeller efficiency in the presence of roughness. Therefore, the required precautions should be regularly taken by ship operators for the propellers. However, it may not be suitable because of the dry dock times, places, and the cost [27]. Instead of cleaning or polishing the propeller, the propeller coating applications can be favourable to minimise or completely prevent the efficiency loss. Since coating can control the fouling growth, it provides a smooth surface condition [28].

Figure 11 shows the non-dimensional pressure contours at different sections of the propeller at $J=0.397$. The great majority of the thrust is commonly generated between the non-dimensional radius of $r / R=0.3$ and $r / R=0.8$ for fixed pitch propellers. Thus, it is appropriate to investigate the impact of the roughness on the pressure distribution of these sections. As the surface roughness accelerates the transition of the laminar boundary layer into the turbulent flow, it results in more frictional losses [33]. The transition of the boundary layer also changes the pressure distributions at all propeller blade sections, which causes a decrease in thrust. As it can be seen in Figure 11, the roughness influences the pressure distributions both at the back (suction) and face (pressure) sides until $r / R=0.7$ which results in the change of the lift and hence thrust generated by the propeller blades. The effects on the pressure distribution are more prominent towards the trailing edge (TE) of the sections starting somewhere from the $1^{\text {st }}$ quarter of the chord for both the suction and pressure sides. This can be attributed to the increasing boundary layer thickness activity toward the tail ends depending upon the shape and location of the maximum thickness of the sections. These latter parameters also reflect on the pressure coefficients of the outer blade sections (i.e. $r / R=0.7-0.8$ ). 

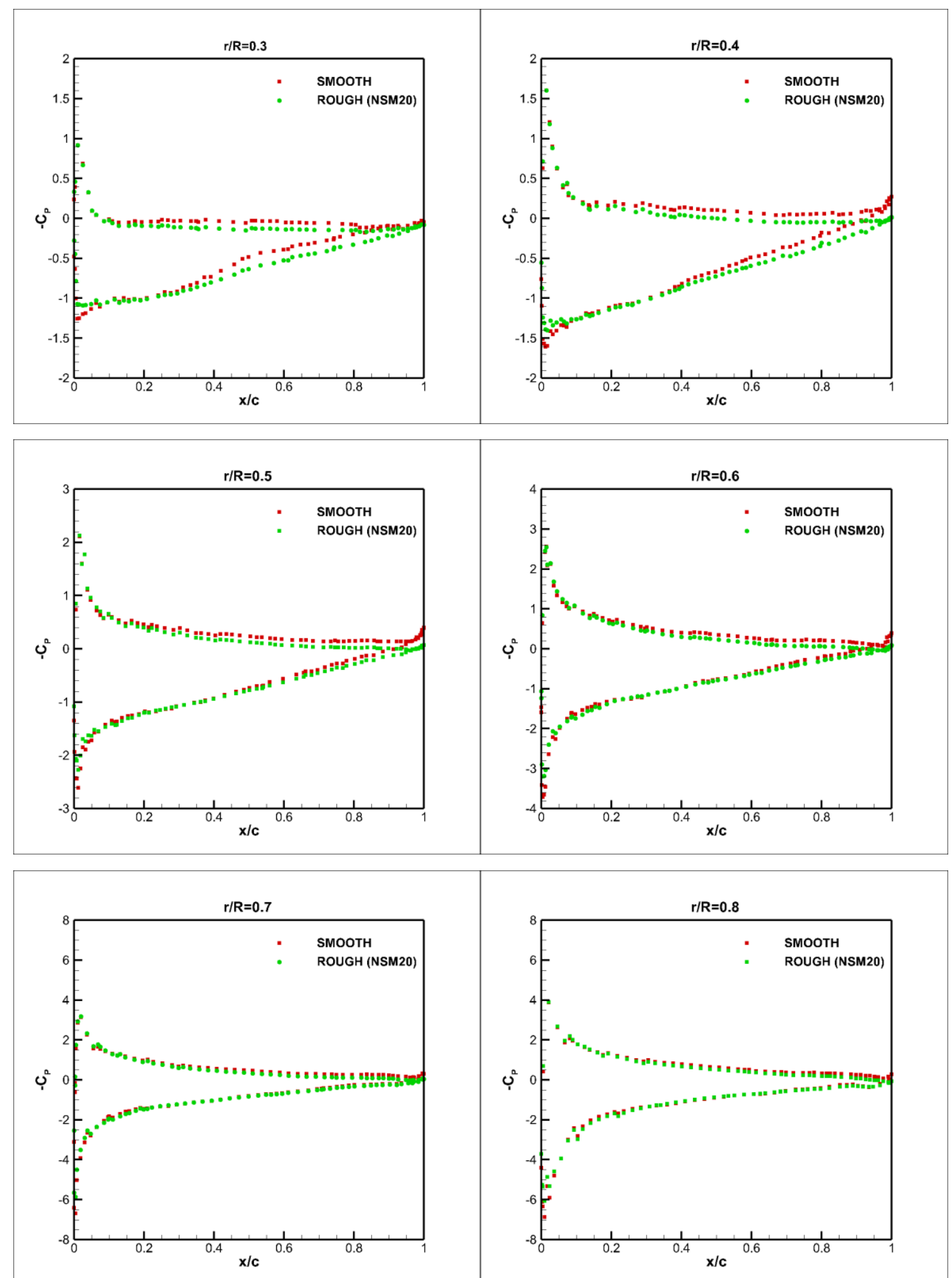

Figure 11. Pressure distributions at different sections at $J=0.397$ 


\subsubsection{Cavitating condition}

\subsubsection{Smooth Case}

In modelling propeller cavitation, types of cavitation (i.e. TVC, sheet, bubble) and their inceptions are critical, and the boundary layer development plays an essential role in predicting and analysing these characteristics, especially for particular types, e.g. TVC. The change of the near-wall grid properties (i.e. boundary layer) influences both propeller performance characteristics (i.e. thrust and torque) and development of cavity bubbles. The prediction of TVC depends on the accurate solution of the flow field inside the vortex core by reducing the numerical diffusion. [69].

As explained in Section 4.3, by implementing the AMR technique locally with a proper resolution of boundary layer properties, the helical structure of the cavitating tip vortex becomes visible at the propeller blade tips. Then, the roll-up phenomenon, which may occur due to the trailing vortex sheet as well as the interaction between the sheet and tip vortex cavitation, can be predicted. During this process, it should be noted that the initial tip vortex evolution and roll-up phenomenon are very sensitive to the boundary layer resolution, especially for the RANS based solvers [79], [80], [81], [69]. Bearing in mind the above background information, for the cavitating propeller in smooth condition, the first grid point was shifted to the viscous sublayer (i.e. around $y^{+}=1$ ) by increasing the number of prism layers for better modelling of the boundary layer (i.e. resolving the boundary layer without wall function). The remaining properties of the grid were kept constant, including the AMR procedure. In this way, the capabilities of the proposed AMR technique can be proven with the experimental observation.

In Figure 12, the results of the simulations for the cavitation patterns of the INSEAN benchmark propeller is shown in comparison with the experimentally observed cavitation patterns for the smooth condition. The comparison suggests that the sheet cavitation was slightly over predicted, while the prediction of the hub vortex cavitation was quite good, including the simulation of the roll-up phenomenon as observed in the experiments. It can be seen in Figure 12 that the accurate prediction of the roll-up phenomenon enables the extension of TVC further downstream. It should also be noted that the more stretched TVC extension can be simulated using more advanced numerical models (e.g. DES or LES). Since the standard RANS models produce a higher amount of turbulent viscosity in the vortex cores, this consequently causes failure to stretch the extension of TVC when compared to advanced numerical models [82]. Nevertheless, the standard RANS models can be considered as a reliable, practical, and more computationally affordable approach for the prediction of TVC with adequate grid and boundary layer properties, particularly in the design stage [69]. 


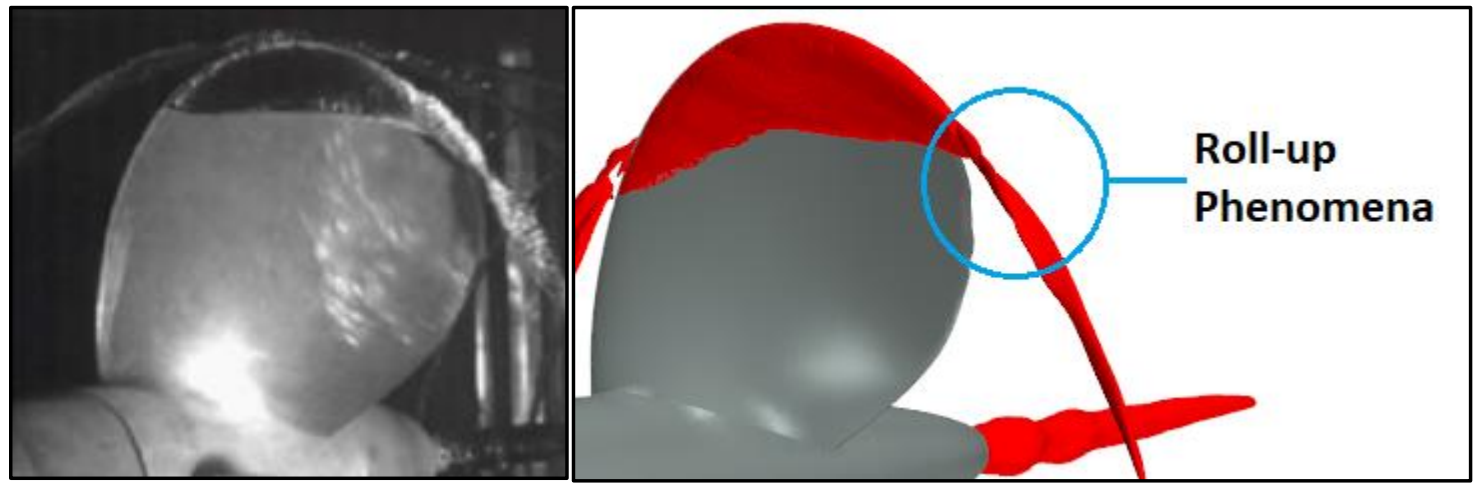

Figure 12. Comparison of cavity shape with the experimental observation ( $\alpha=0.1, J=0.71, \sigma=1.76)$

\subsubsection{Rough Case}

The strength and fluid properties of vortex core (such as velocity and pressure) are determined by close interaction of boundary layers on the suction and pressure sides of the propeller in addition to trailing vortices [33]. As stated above, the evolution of tip vortex formation strongly depends on the boundary layer development on the propeller blades [80], [79], [81], [69]. Thus, the flow around the smooth propeller was resolved again using the determined near wall-properties (i.e. $y^{+}>k^{+}$) to make a fair comparison between smooth and rough cases. For this reason, the number of prism layer was decreased, and the average $y^{+}$value was tuned from $y^{+}=1$ to around $y^{+}=280$ to satisfy the criteria of wall function model (i.e. $y^{+}>k^{+}$) in smooth condition by taking the biggest roughness height into account. The reason behind this was to show the effects of roughness on the cavitation phenomenon, especially for TVC, since the boundary layer modelling is considerably important for the initial formation of TVC and its extension. It must be born in mind that the $y^{+}=280$ was used in the smooth condition for uncertainty study for cavitation and noise simulations in Section 5.1. Hereafter, the simulations were conducted by using the near-wall properties (i.e. $y^{+}=280$ ) for cavitation and hydroacoustic part unless it is specified.

Table 9 shows the validation of the performance characteristics of the benchmark propeller under the cavitating conditions in comparison with the experimental data in smooth condition. The influence of the roughness on the propeller hydrodynamic performance is also given in the same table. As shown in Table 9, the difference between the CFD and experimental was found around 5\% for thrust and torque coefficients.

As far as the effect of the roughness is concerned, similar to the non-cavitating case, the roughness has a degradation impact on the propeller performance as such the thrust coefficient reduces with increasing roughness while the torque coefficient increases. 
Table 9. Validation and influence of roughness on the propeller of propeller performance characteristics $(J=0.71, \sigma=1.763)$

\begin{tabular}{cccc}
\hline Roughness Type & $K_{T}$ & $10 K_{Q}$ & $\eta_{0}$ \\
\hline Experiment (Smooth) & 0.255 & 0.460 & 0.626 \\
SMOOTH $\left(y^{+} \cong 1\right)$ & 0.242 & 0.438 & 0.624 \\
SMOOTH $\left(y^{+}=280\right)$ & 0.238 & 0.434 & 0.619 \\
M10 & 0.214 & 0.467 & 0.517 \\
NSM10 & 0.210 & 0.474 & 0.502 \\
M20 & 0.202 & 0.491 & 0.464 \\
NSM20 & 0.201 & 0.497 & 0.458 \\
\hline
\end{tabular}

Figure 13 shows the cavitation patterns, both in the smooth and rough conditions. As stated in the previous chapter, the change of cavitation pattern in the smooth case (top sub-figure of Figure 14) in comparison to Figure 12 can be considered due to the different boundary layer resolution. The cavitating volume decreases since the tip vortex, and sheet cavitation become thinner for the rough propellers in comparison to the smooth propeller. Since the roughness stimulates the transition of the boundary layer from laminar to turbulent and changes the near-wall flow structures, it leads to the deterioration of the tip vortex strength. The turbulence triggered by the roughness can destabilise the tip vortex formation and causes its breakdown, which consequently results in a decrease of TVC in comparison to a smooth condition. Having said that the roughness on the propeller blades also causes a slight decrease of the sheet and hub vortex cavitation. As can be clearly seen in Figure 13, with the increase of the roughness heights, the cavitation volume reduces mainly due to the reduction of the TVC in Figure 13. 

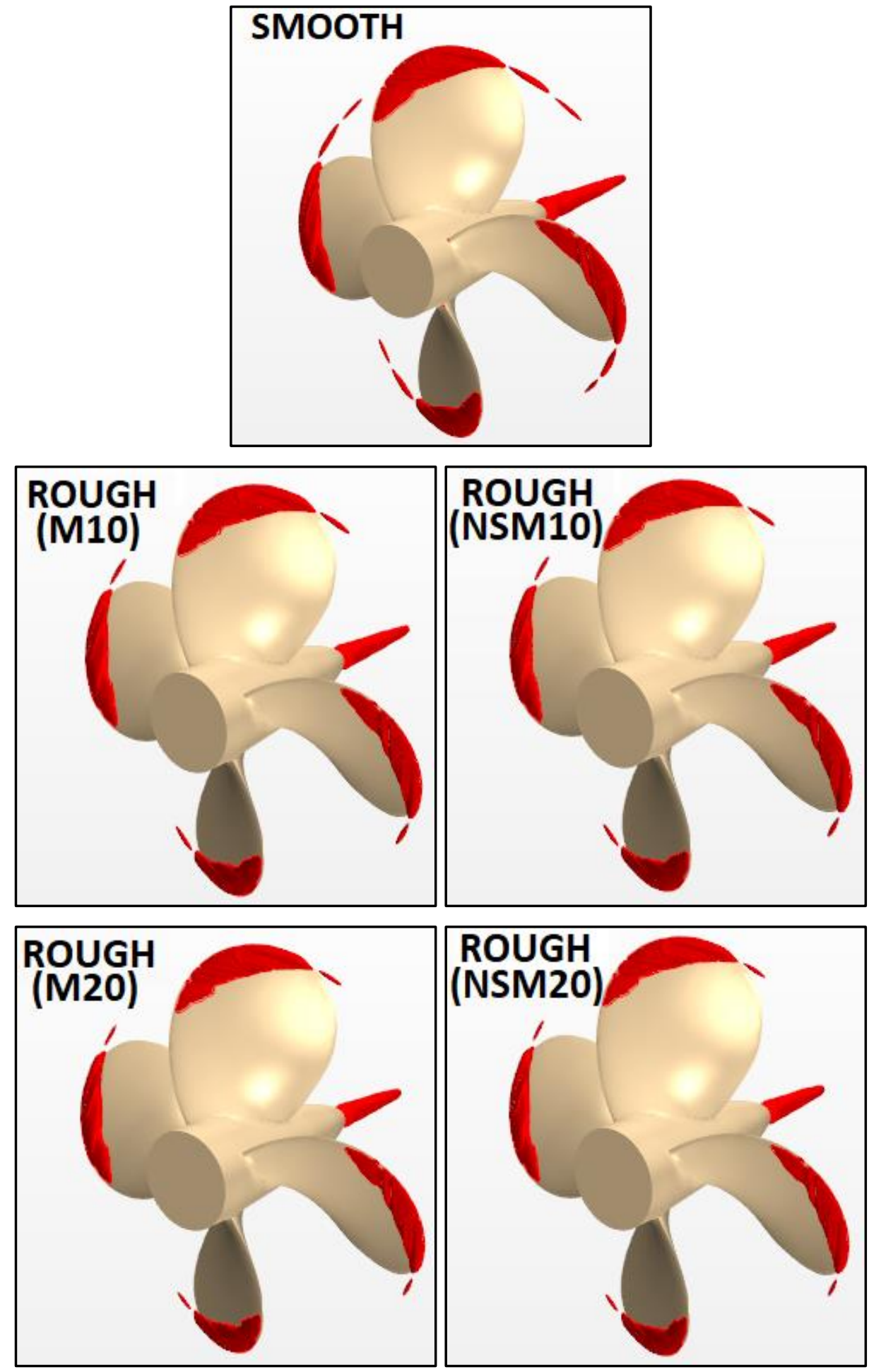

Volume Fraction of Vapor

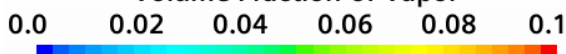

Figure 13. The change of sheet, hub, and tip vortex cavitation with different roughness conditions at $J=0.71$ and $\sigma=1.763$ 
Figure 14 shows the change of vorticity and velocity distribution in the propeller's slipstream both in the smooth and rough conditions. The roughness has considerable influence on the vortex strength and velocity field. Due to the additional viscous stresses between the roughened tip surface and tip vortex, the circumferential momentum of the vortex structure is converted to turbulence kinetic energy. Hence, turbulence kinetic energy and dissipation rate considerably increases due to the roughness [32]. Consequently, the significant amount of vorticity in the propeller's slipstream disappear with an increase of the roughness height. Besides, due to the considerable momentum loss stem from the roughness, the pressure inside the vortex core increases.

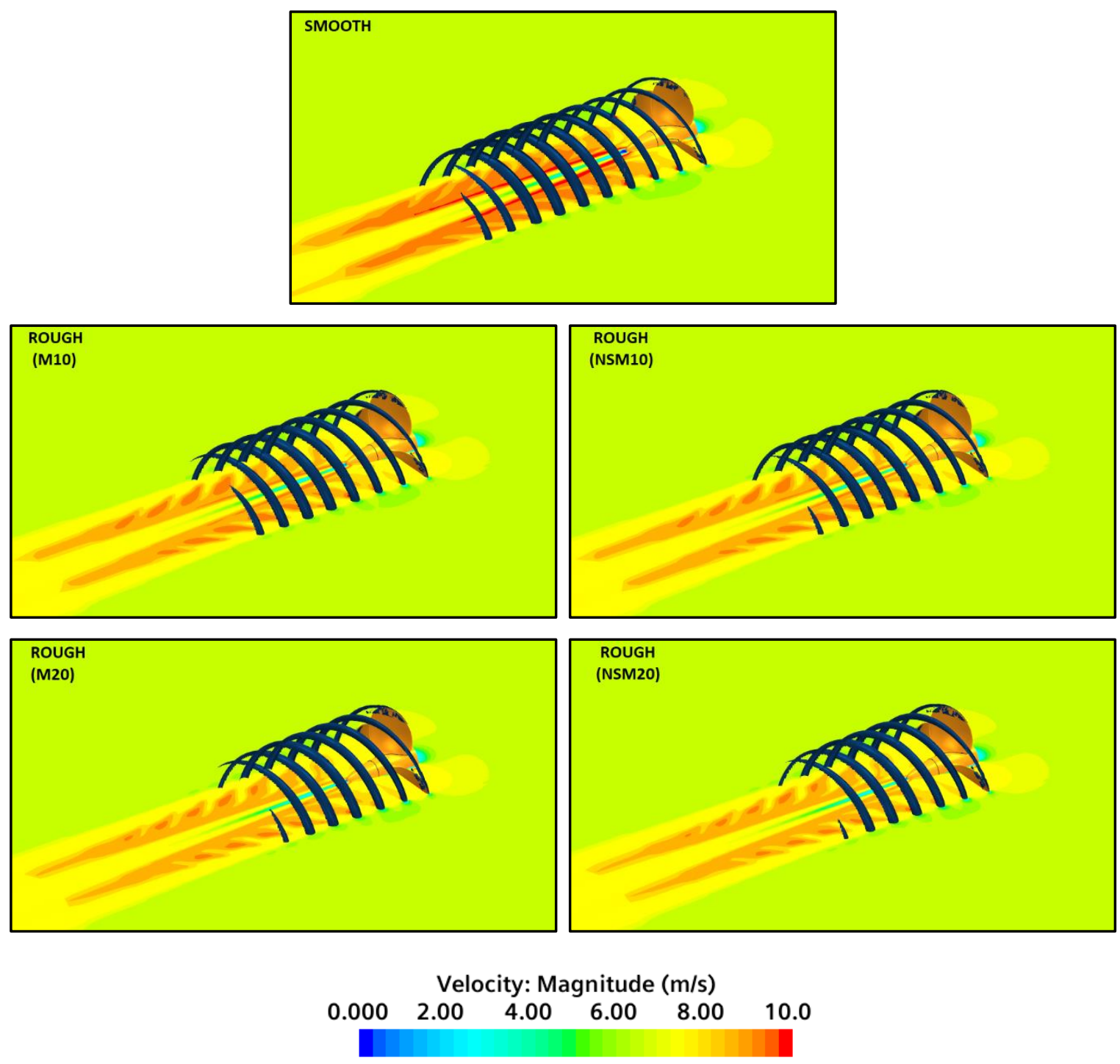

Figure 14. The change of vorticity and velocity fields with different roughness conditions at $J=0.71$ and $\sigma=1.763\left(Q_{c}=40001 / s^{2}\right)$ 


\subsection{Hydroacoustic Results}

\subsubsection{Cavitating Condition}

\subsubsection{Smooth Case}

Following the hydrodynamic simulations, the next is the simulation of the hydroacoustic performance of the benchmark propeller both in the smooth and rough conditions. For this purpose, as described earlier in Section 3.2, a hybrid approach, where the P-FWH equation, which is a state-of-the-art hydroacoustic analogy tool coupled with the CFD-based hydrodynamic solver, is used. The equation has its origin in the aeronautical field, but its use has been recently spread in the maritime community for the marine applications (e.g. [83], [52], [84], [85]).

As explained in Section 3.2, the porous formulation of FW-H equation, which is a relatively new formulation, is utilised to predict the propeller URN. In this approach, the integral domain is placed around the propeller region, which encompasses relevant non-linear noise sources which are mainly represented by vorticity and turbulence-induced in propeller's slipstream. In this way, the non-linear effects can be included in the calculations since their contribution can be significant in the presence of cavitation, especially for the far-field noise estimations.

In the hydroacoustic simulations, the acoustic pressures are computed in the time-domain on the selected noise surface and transferred to the frequency domain by using the Fast Fourier Transform for each receiver. Then, the Sound Pressure Level (SPL) can be calculated using the following equation.

$$
S P L=20 \log \left(\frac{p}{p_{\text {ref }}}\right)
$$

Here, $p$ is the acoustic pressure; $\mathrm{Pa}, p_{\text {ref }}$ is the reference pressure $(1 \mu)$ for water; $\mathrm{Pa}$.

Figure 15 shows the integral noise surface around the benchmark propeller, which is extended to around 3D downstream along the propeller slipstream to cover the relevant noise sources as much as possible. This surface should be located inside the fine grid region to calculate direct hydroacoustic pressure fluctuations from the noise sources such as vortices and cavitation bubbles to avoid any risk of information loss [84]. Otherwise, i.e., if the noise surface is located far away from the propeller, where the vorticity field may still exist, and in relatively coarse grid region, the prediction of the URN results would be unreliable. This inaccuracy is due to the numerical noises which are attributed to the sliding interfaces and numerical dissipation related to the grid resolution. Nevertheless, there is no practical guideline for the integral surface location and dimensions in the maritime field and still open to discussion in the literature [52], [83]. 


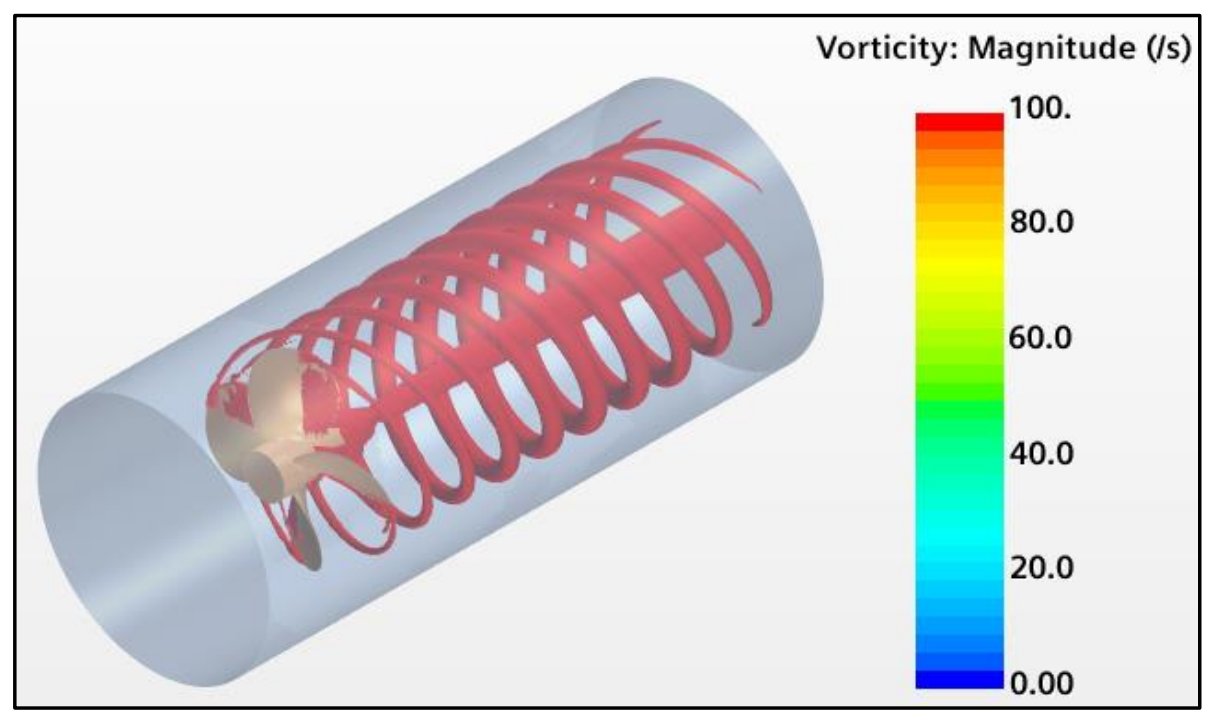

Figure 15. The representation of integral surface for URN predictions $\left(Q_{c}=40001 / s^{2}\right)$

The hybrid approach used for the hydroacoustic prediction of the benchmark propeller can be considered as the post-processing of the hydrodynamic solution using the acoustic analogy, which provides the evaluation of the URN in the far-field. Since there is no available experimental noise data for the selected benchmark propeller, the consistency of the numerical solution can be proved by a comparison of the hydrodynamic pressures obtained directly solving the Navier-Stokes equations and the hydroacoustic pressures provided by acoustic analogy in the near field. Thus, the reliability of the numerical solution gives confidence for the accurate evaluation of the URN in the far-field.

In order to conduct the above-mentioned comparison, Figure 16 and Table 10 are shown the representation and location of the receivers in the near field, respectively. Receiver 2 (R2) is located at the propeller plane, whereas receiver 1 (R1) and receiver 3 (R3) are located both upstream and downstream, respectively.

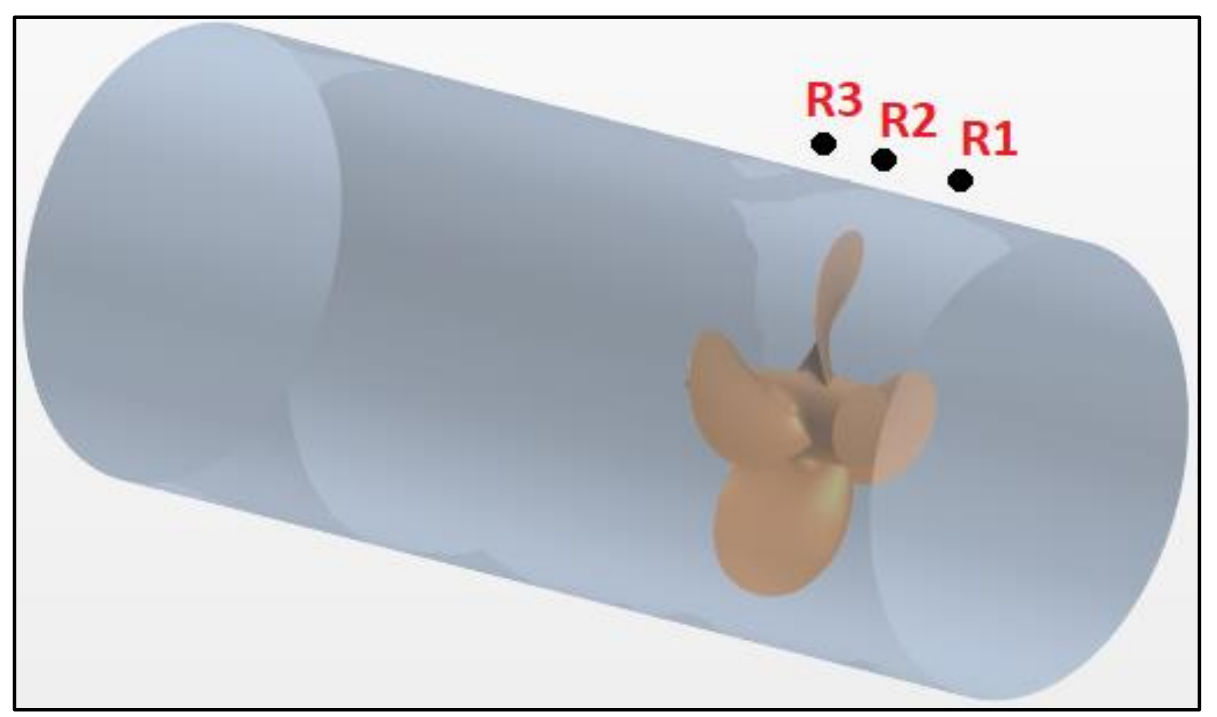

Figure 16. Representation of near-field receivers (Figure is not scaled) 
Table 10. Location of the near-field receivers

\begin{tabular}{cccc}
\hline Receiver & $X(\mathrm{~m})$ & $\mathrm{Y}(\mathrm{m})$ & $\mathrm{Z}(\mathrm{m})$ \\
\hline 1 & 0.06 & 0 & 0.1704 \\
2 & 0 & 0 & 0.1704 \\
3 & -0.04 & 0 & 0.1704 \\
\hline
\end{tabular}

The verification of the near-field pressures is shown in Figure 17 by the comparison of the predicted pressures both by the hydrodynamic solver, and the hydroacoustic analogy. Since the receivers are located in the vicinity of the propeller, the overall shape of the signal is smooth and purely characterised by the Blade Passage Frequency (BPF) (i.e. four peaks associated with the four blades). Besides, the contribution of the monopole and dipole (linear) noise terms of the $\mathrm{FW}-\mathrm{H}$ equation is higher than the quadrupole (i.e. non-linear) noise term for the near-field receivers. The agreement between the hydrodynamic (RANS based) pressures, and the porous $\mathrm{FW}-\mathrm{H}$ based pressures is quite good at three different locations. As expected at R2, which is located at the propeller plane, the pressure fluctuations are higher than the remaining receiver locations since the blade harmonics are more dominant. In this receiver location, the hydrodynamic (RANS based) pressure might be used to evaluate the hydroacoustic performance of the propeller at this operating condition. However, as one moves far away from the propeller, the hydrodynamic pressures tend to suffer from numerical dissipation. Hence, the acoustic analogy by the $\mathrm{FW}-\mathrm{H}$ equation is needed to yield better noise estimation in the far-field. 

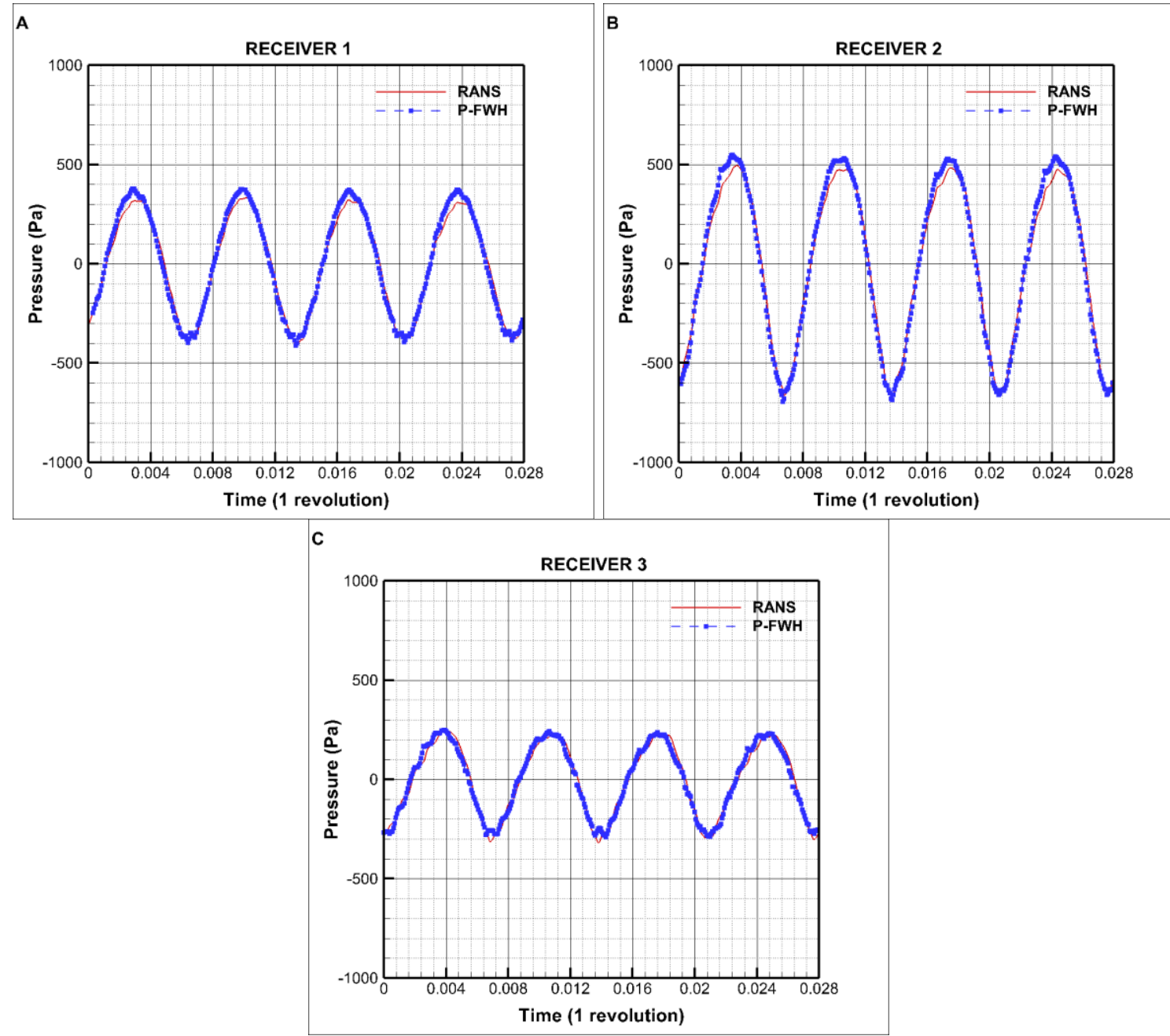

Figure 17. Verification of hydrodynamic and hydroacoustic pressures in the near field

\subsubsection{Rough case}

Having conducted the simulations for the smooth case in cavitating condition, this Section presents the simulations for the rough propeller case in cavitating condition.

As it was studied in Section 5.2.2, the hydrodynamic simulations with the rough propeller under the cavitating conditions displayed a significant change in the cavitation volume and maximum change was observed with the NSM20 roughness type, which also has the maximum roughness height. Therefore, the CFD simulations are conducted for the NSM20 roughness type, and its effects on the propeller URN are explored both in the near and far-fields.

As it is well-known, the frequency range of the noise source contributions into the URN spectrum of a marine propeller can be broadly investigated at two levels: low frequency and high-frequency (or broadband) levels. In the low-frequency level of the noise spectrum, the major contributions are from the monopole and dipole sources, which are associated with the thickness, pressure change and cavity volume characteristics of the blades which manifest themselves in the discrete blade rate frequencies (or spectral peaks) of the spectrum. In contrast, the broadband range of the URN spectrum is 
contributed by the quadruple sources, which are associated with the vorticity, turbulence and its interaction with the wall boundary layer [78] as well as the cavitation dynamics especially with the cavity collapses and vortex interaction. Since the boundary layer is one of the crucial parameters affecting the formation of the turbulence, vorticity and cavity bubbles, it is expected that the roughness will also impact on the URN characteristics due to the inherent relationship between the roughness and the boundary layer.

Figure 18 shows the effect of the NSM20 type roughness on the benchmark propeller's URN (acoustic pressure) levels predicted at the three receivers, which are located in the near field as listed in Table 8 under cavitating condition. The comparison of the predicted acoustic pressure levels for the smooth and rough blades in the near field shows that there are no discernible differences in terms of the overall amplitude, although, the pressure amplitudes for the rough case are very slightly higher. The predicted acoustic pressures are smooth and characterised by the BPF in both cases. As stated earlier, the overall acoustic signature in the near field is dominated by the blade harmonics (i.e. from the monopole and dipole sources) while the contribution from the quadrupole sources is not expected to be felt in the vicinity of the propeller blades and hub.

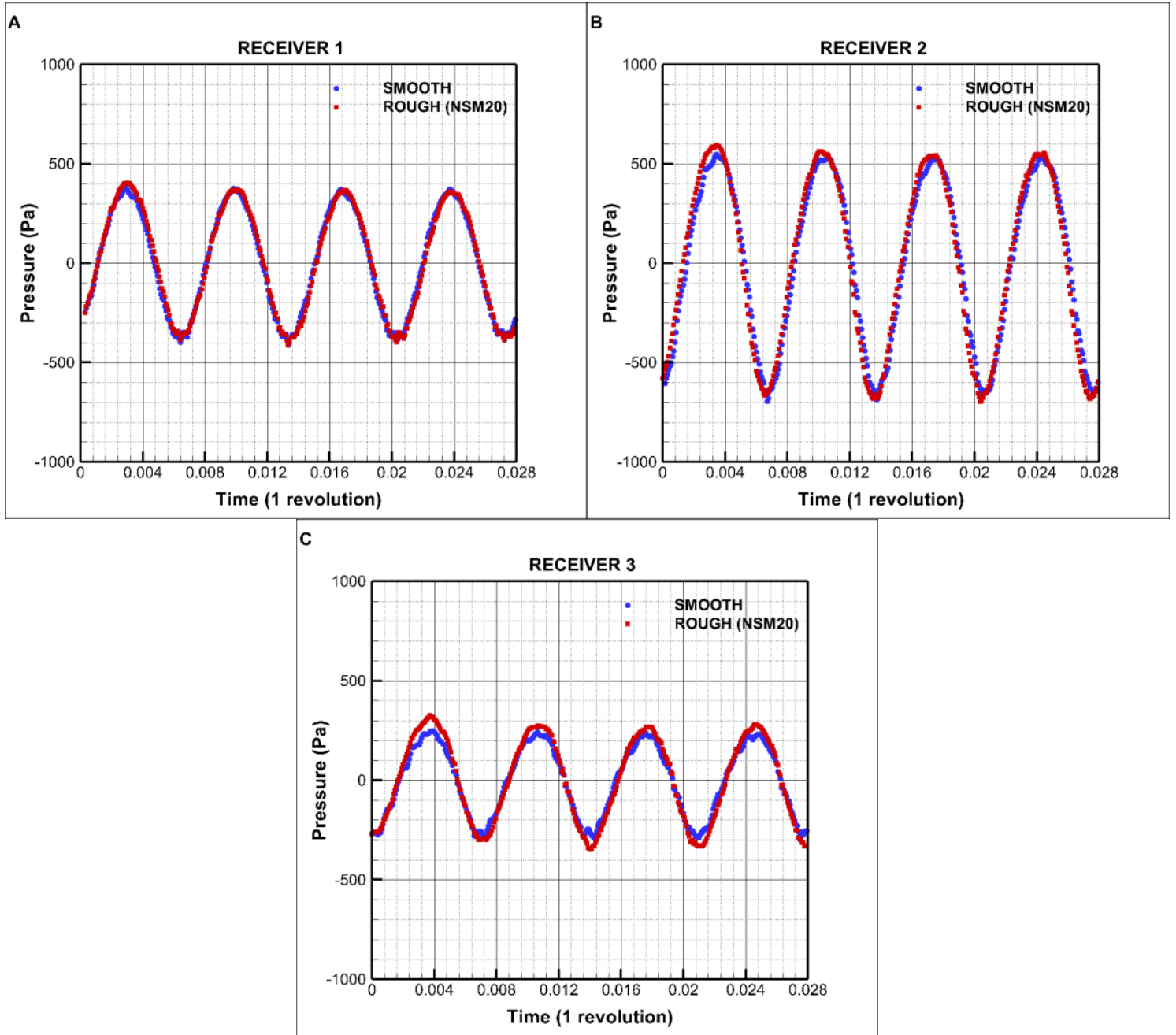

Figure 18. Comparison of acoustic pressures both in smooth and rough conditions in the near field 
The acoustic pressure waves have a different impact in the near and far-fields depending upon the location of the receivers and contribution of the noise sources. The effects of the non-linear noise terms become dominant when the receivers are located in the far-field. Thus, the changes in the boundary layer properties and the cavitation dynamics induced by the roughness are felt and can be analysed more effectively using the frequency spectra at the narrow band in the far-field. By considering the standard procedure of using $1 \mathrm{~m}$ distance from the propellers as the reference distance, (e.g. recommended by ITTC), the three receivers can be located at a distance of $1 \mathrm{~m}$ (i.e. around 4.4D), 5.675m (i.e. approximately 25D) and $22.7 \mathrm{~m}$ (i.e. around 100D) away from the intersection point of the propeller plane with the shaft centre. In this way, the effects of roughness on the propeller URN levels can be investigated in the far-field.

Based on the above-described arrangement of the receivers and using the hybrid approach, the hydroacoustic simulations are conducted with the benchmark propeller, and results are presented in the narrowband, as shown in Figure 19. By concentrating on the broadband frequency range of the spectrum up to $6.5 \mathrm{kHz}$, Figure 19 indicates that the effect of roughness decreases the acoustic pressure levels between the $1 \mathrm{kHz}-2 \mathrm{kHz}$ range regardless of the receiver locations (see zoomed zones in Figure 19). In this frequency interval, the noise levels decrease up to approximately $10 \mathrm{~dB}$. Above $2 \mathrm{kHz}$, the effect of the roughness appears to diminish by display the almost similar backbone curves of sound pressure levels for the smooth and rough blades but with the oscillating amplitudes. The change in trend after $2 \mathrm{kHz}$ can be associated with the increased turbulence activity, which results in the reduced cavitation volume (mainly due to TVC) and associated dynamics as discussed earlier in Section 5.2.2.2 for the effect of roughness on cavitation. It should be noted that the noise levels increased below $100 \mathrm{~Hz}$ in the presence of roughness. The reason being might be due to the short FFT time interval for the low-frequency range below $100 \mathrm{~Hz}$. Nevertheless, it needs to be investigated with further studies. 


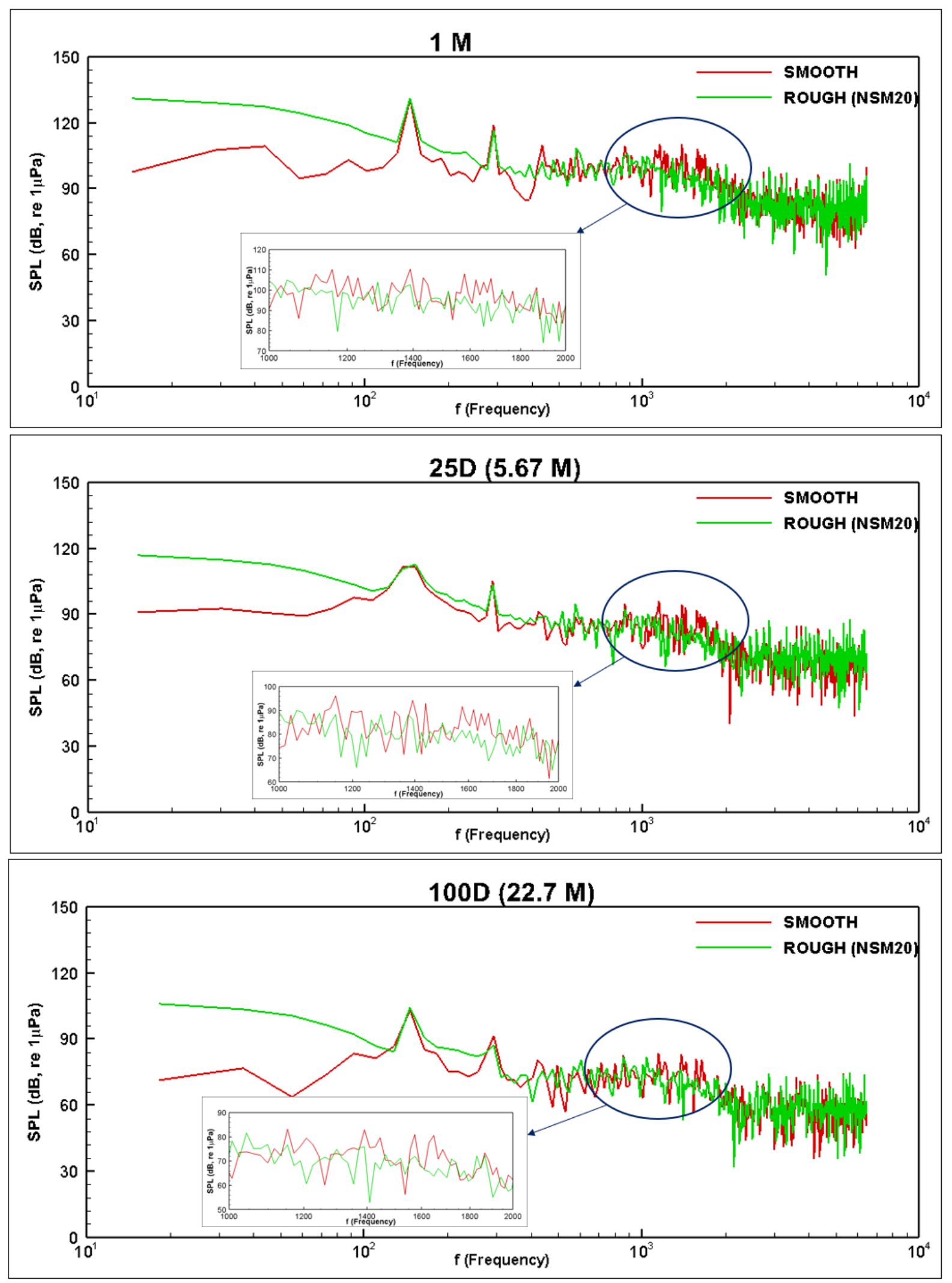

Figure 19. The influence of roughness on propeller URN in the far-field 
The variation of the sound pressure levels with the blade passing frequencies (BPF) for the smooth and rough propeller blades is shown in Figure $\mathbf{2 0}$ for three different receiver locations in the far-field. The noise levels at the BPFs include a contribution from the non-cavitating source as well as the cavitating source. The former is related to the fluctuating blade loading and blade thickness, while the latter is associated with the sheet cavitation volume variations, acting as a monopole source. As can be seen in Figure 20 , whereas the noise level at the $1^{\text {st }}$ BPF is not affected by the roughness significantly, the noise levels at the $2^{\text {nd }}$ and $3^{\text {rd }}$ BPFs reduce with the roughness between 1-7 $\mathrm{dB}$ which can be associated with the change in cavity volume variations. However, this trend reverses at the $4^{\text {th }}$ BPF value with slightly increased noise levels in the rough condition.

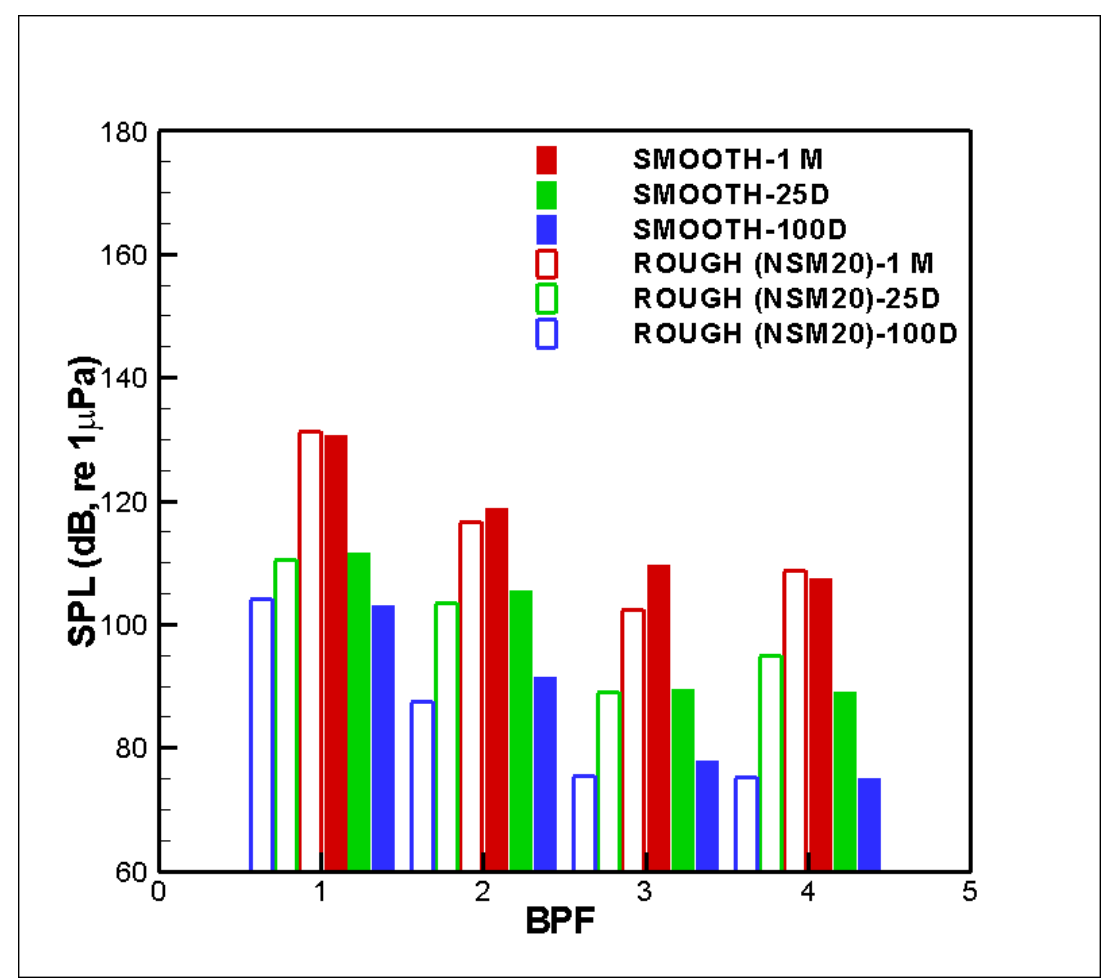

Figure 20. The change in BPF values with roughness

For the benchmark propeller, the simulation results indicate that the roughness has a favourable reduction effect on the noise levels, particularly between the $1 \mathrm{kHz}$ and $2 \mathrm{kHz}$ range and at the $2^{\text {nd }}, 3^{\text {rd }}$ BPFs in the uniform flow conditions. However, as stated in Table 7, the roughness also reduces the propeller efficiency at around $25 \%$. This finding, therefore, suggests that there should be a compromise between the two conflicting design requirements (i.e. hydrodynamic and hydroacoustic performance optimisations) for marine propellers. The deteriorating impact of roughness on the propeller hydrodynamic performance can be diminished by applying the roughness on small strategic areas of the propeller blades. Notably, as stated in the study of Philipp and Ninnemann [37] and Kruger et al. [32], it can be only applied at the propeller blade tips, where the tip vortex forms, to reduce the tip loading, hence the TVC. In this way, the cavitation volume decreases, whereas the propeller efficiency can be kept as high as possible close the smooth condition. In a very recent study of Asnaghi 
et al. [35], the roughness was applied at the tip of the elliptical foil, and those authors managed to prevent the performance degradation (i.e. lift and torque) by reducing the TVC. Therefore, akin to the other noise mitigation methodologies such as PressurePores ${ }^{\mathrm{TM}}$ [86], modification of the propeller blade geometries [87], the roughness applications on the small and strategic areas of the propeller blade tips can be an effective way for mitigation of the propeller URN for retrofitting as well as new design projects.

\subsection{Discussion}

As stated in Section 1 (Introduction part) of the paper, this study is a step forward to explore the impact of the surface roughness, in particular biofouling related roughness, on a marine propeller's hydrodynamic and hydroacoustic performance. Within this context, the study has concentrated on the roughness effect, particularly, on the cavitation and URN performance of propellers operating in isolation (i.e. in open water without any wake) and using a limited amount of validation data which is hard to obtain, especially for the URN. As it is a well-known fact that the effect of the hull wake on the propeller performance is essential not only for the propulsive efficiency but also for the cavitation, vibration and URN. Application of roughness is, therefore, important to include the wake nonuniformity on the simulations as well as the scale effects by conducting the simulations in full-scale. When one considers including the effect of the hull wake on the propulsive performance in the presence of roughness, the roughness will not only affect the propeller's performance through the propeller open water efficiency (as explored in this paper). It will also affect the propeller-hull interaction coefficients through its effect on the wake. The impact of the roughness on the hull wake will change the propeller advance coefficient, which in turn affect the propeller efficiency as well as the hull and relative-rotative efficiencies. Consequently, the propeller's cavitation inception and cavitation patterns; propeller induced hull vibrations and the URN of the propeller will be affected.

The effect of biofouling related roughness on a representative full-scale container vessel, KCS, was recently presented by Song et al. [5] by using the CFD and associated wall-function procedure, similar to the one used in this study. Their study is significant by demonstrating the effect of the biofouling related roughness on a self-propelling vessel in full-scale. However, still, it explored the roughness effect on the propulsive performance by neglecting the effect of cavitation, and no consideration was given to the URN. It is, therefore, critical that the present paper is a further step forward to simulate the cavitation and URN performance in the presence of the roughness. While the present study, so far, presented the results for the open water propellers in model scale, the authors have been expanding their research to address the effects of roughness on a self-propelled vessel in full-scale. 


\section{CONCLUSION}

In this study, the effects of biofouling-related roughness on the hydrodynamic and hydroacoustic performance of a marine propeller were investigated in model-scale and open water condition by using a CFD model. The study presented the effect of a particular biofouling roughness on the URN levels of a propeller at model-scale and using the CFD simulations for the first time.

Results showed that with an increasing roughness height, the propeller thrust decreases while the torque increases. Thus, the propeller efficiency decreases between $8 \%$ and $30 \%$ depending on the roughness height and configuration, under both non-cavitating and cavitating conditions. Consequently, it was found that the roughness has a negative impact on the propeller hydrodynamic performance.

On the other hand, the roughness showed some positive effects on the reduction (and mitigation) of the cavitation and the level of the propeller URN. In the presence of the roughness, cavitation volume (particularly the TVC) reduced, hence the level of the propeller URN. No discernible difference was observed between the smooth and rough blade cases of the propeller URN levels at the receivers located in the near field. In contrast, the URN levels decreased up to approximately $10 \mathrm{~dB}$ depending on the distance between $1 \mathrm{kHz}$ and $2 \mathrm{kHZ}$. Also, the URN levels at the $2^{\text {nd }}$ and $3^{\text {rd }} B P F$ decreased from 1 to $7 \mathrm{~dB}$ depending on the distance.

In this study, it is important to note that uniform flow condition was presumed into the propeller. However, the mitigation of tip vortex cavitation is particularly more important in the presence of the non-uniform wake field. Thus, the current study is being further expanded to investigate the effects of roughness in a complete model by taking hull, rudder, etc. into account. Besides, the roughness application on the strategic areas of the propeller blades is currently being investigated at several operational points under cavitating conditions. The aim is here to prevent propeller efficiency loss as much as possible while still reducing cavitation volume.

On a final note, since the current CFD approach is mainly associated with the barnacle-type biofouling in different sizes, it can also be extended to investigate the other physical roughness types, e.g. hull roughness due to welding, corrosions, coating, slime etc. e.g. Yeginbayeva et al. [3], using the proposed CFD approach in the future.

\section{ACKNOWLEDGEMENT}

The first author is sponsored by the Stone Marine Propulsion Ltd of the UK and the University of Strathclyde during his PhD study. The Turkish Ministry of Education funds Mr Refik Ozyurt's doctoral study. Also, the granted access to the High-Performance Computing for the West of Scotland (Archie- 
West) is acknowledged. The authors are also grateful to CNR-INSEAN, especially to Francesco Salvatore, for providing the propeller geometry and experimental results.

\section{REFERENCES}

[1] M.P. Schultz, Effects of coating roughness and biofouling on ship resistance and powering, Biofouling. 23 (2007) 331-341. https://doi.org/10.1080/08927010701461974.

[2] M.P. Schultz, J.A. Bendick, E.R. Holm, W.M. Hertel, Economic impact of biofouling on a naval surface ship, Biofouling. 27 (2011) 87-98. https://doi.org/10.1080/08927014.2010.542809.

[3] I.A. Yeginbayeva, M. Atlar, An experimental investigation into the surface and hydrodynamic characteristics of marine coatings with mimicked hull roughness ranges, Biofouling. 34 (2018) 1001-1019. https://doi.org/10.1080/08927014.2018.1529760.

[4] N. Speranza, B. Kidd, M.P. Schultz, I.M. Viola, Modelling of hull roughness, Ocean Eng. 174 (2019) 31-42. https://doi.org/10.1016/j.oceaneng.2019.01.033.

[5] S. Song, Y.K. Demirel, M. Atlar, Penalty of hull and propeller fouling on ship self-propulsion $\begin{array}{llllll}\text { performance, Appl. Ocean } & \text { Res. } & 94 & \text { (2020) }\end{array}$ https://doi.org/10.1016/j.apor.2019.102006.

[6] Y.K. Demirel, D. Uzun, Y. Zhang, H.-C. Fang, A.H. Day, O. Turan, Effect of barnacle fouling on ship resistance and powering, Biofouling. 33 (2017) 819-834. https://doi.org/10.1080/08927014.2017.1373279.

[7] D. Uzun, Y.K. Demirel, A. Coraddu, O. Turan, Time-dependent biofouling growth model for predicting the effects of biofouling on ship resistance and powering, Ocean Eng. 191 (2019) 106432. https://doi.org/10.1016/j.oceaneng.2019.106432.

[8] D. Owen, Y.K. Demirel, E. Oguz, T. Tezdogan, A. Incecik, Investigating the effect of biofouling on propeller characteristics using CFD, Ocean Eng. 159 (2018) 505-516. https://doi.org/10.1016/j.oceaneng.2018.01.087.

[9] ABS, Ship energy efficiency measures advisory. American Bureau of Shipping Houston, 2013.

[10] ITTC, Performance, Propulsion 1978 ITTC Performance Prediction Method, ITTC Recommended Procedures, 2011.

[11] N.D. Merchant, P. Blondel, D.T. Dakin, J. Dorocicz, Averaging underwater noise levels for environmental assessment of shipping, J. Acoust. Soc. Am. 132 (2012) EL343-EL349. https://doi.org/10.1121/1.4754429.

[12] J.A. Hildebrand, Anthropogenic and natural sources of ambient noise in the ocean, Mar. Ecol. Prog. Ser. 395 (2009) 5-20.

[13] R.J. Urick, Principles of underwater sound 3rd edition, Penins. Publising Los Atlos, Calif. 22 (1983) 23-24.

[14] P. Kellett, O. Turan, A. Incecik, A study of numerical ship underwater noise prediction, Ocean Eng. 66 (2013) 113-120. https://doi.org/10.1016/j.oceaneng.2013.04.006.

[15] IMO, MEPC.1/Circ.833: Guidelines for the Reduction of Underwater Noise from Commercial Shipping to Address Adverse Impacts on Marine Life, 44 (2014) 8.

[16] IWC, Report of the scientific committee, in: J. Cetacean Res. Manag., 2009: pp. 46-47.

[17] SONIC, Suppression Of underwater Noise Induced by Cavitation | SONIC Project | FP7 | CORDIS | European Commission, in: EC-FP7, Grant Agreem. No 2012314394.

[18] AQUO, Achieve QUieter Oceans by shipping noise footprint reduction | AQUO Project | FP7 | CORDIS | European Commission, in: Grant Agreem. No 314227.

[19] SILENV, Ships oriented Innovative soLutions to rEduce Noise \& Vibrations | SILENV Project | FP7 | CORDIS | European Commission, in: 7th Framew. Program, Grant Agreem. No 234182.

[20] 28th ITTC The Specialist Committee on Hydrodynamic Noise Final Report and Recommendations, 2017.

[21] History of HTF | Hydro Testing Forum, (n.d.). https://www.hydrotestingforum.org/history-of- 
htf.

[22] A. Milne, Roughness and drag from the marine paint chemist's viewpoint, in: Mar. Rough. Drag Work. Pap., 1990.

[23] Y.K. Demirel, M. Khorasanchi, O. Turan, A. Incecik, On the importance of antifouling coatings regarding ship resistance and powering, in: Low Carbon Shipp. Conf., London, 2013: pp. 1-13.

[24] Y.K. Demirel, O. Turan, A. Incecik, Predicting the effect of biofouling on ship resistance using CFD, Appl. Ocean Res. 62 (2017) 100-118. https://doi.org/10.1016/j.apor.2016.12.003.

[25] D. Uzun, R. Ozyurt, Y.K. Demirel, O. Turan, Does the barnacle settlement pattern affect ship resistance and powering?, Appl. Ocean Res. $95 \quad$ (2020) 102020. https://doi.org/10.1016/j.apor.2019.102020.

[26] R.L. Townsin, D. Byrne, T.E. Svensen, A. Milne, Estimating the technical and economic penalties of hull and propeller roughness, Trans. SNAME. 89 (1981) 295-318.

[27] M.A.A.-R. Mosaad, Marine propeller roughness penalties, University of Newcastle upon Tyne, 1986.

[28] M. Atlar, E.J. Glover, M. Candries, R.J. Mutton, C.D. Anderson, The effect of a foul release coating on propeller performance, in: Int. Conf. Mar. Sci. Technol. Environ. Sustain. (ENSUS 2002), University of Newcastle upon Tyne, 2002.

[29] M. Atlar, R. Mutton, E.J. Glover, C.D. Anderson, Calculation of the effects of new generation coatings on high speed propeller performance, in: 2nd Int. Warsh. Cathodic Prot. Symp. Equip. Exhib., Newcastle University, 2003.

[30] C. Anderson, M. Atlar, M. Callow, M. Candries, A. Milne, R. Townsin, The development of foulrelease coatings for seagoing vessels, Proc. Inst. Mar. Eng. Sci. Technol. Part B J. Mar. Des. Oper. 4 (2003) 11-23.

[31] K.-C. Seo, M. Atlar, B. Goo, A Study on the Hydrodynamic Effect of Biofouling on Marine Propeller, J. Korean Soc. Mar. Environ. Saf. 22 (2016) 123-128. https://doi.org/10.7837/kosomes.2016.22.1.123.

[32] C. Krüger, N. Kornev, L. Greitsch, Influence of propeller tip roughness on tip vortex strength and propeller performance, Sh. Technol. Res. 63 (2016) 110-120. https://doi.org/10.1080/09377255.2016.1205293.

[33] Abolfazl Asnaghi, Robert Gustafsson, Rickard E. Bensow, Urban Svennberg, Roughness Effects on the Tip Vortex Strength and Cavitation Inception, Sixth Int. Symp. Mar. Propulsors. (2019).

[34] S. Song, Y.K. Demirel, M. Atlar, An investigation into the effect of biofouling on full-scale propeller performance using CFD, in: Proc. Int. Conf. Offshore Mech. Arct. Eng. - OMAE, American Society of Mechanical Engineers (ASME), 2019. https://doi.org/10.1115/OMAE201995315.

[35] A. Asnaghi, U. Svennberg, R. Gustafsson, R.E. Bensow, Investigations of tip vortex mitigation by using roughness, Phys. Fluids. 32 (2020) 065111. https://doi.org/10.1063/5.0009622.

[36] J. Van der Kooij, Sound Generation by Bubble Cavitation on Ship Propellers: The effects of Leading Edge Roughness, in: ASME, 2nd Int. Symp. Cavitation Multiph. Flow., 1986.

[37] O. Philipp, P. Ninnemann, Wirkung von Fluegelrauigkeiten auf Kavitation und Erregung, Maritime Speech Day, Technical University Hamburg Harburg, in: 2007.

[38] W.W. Cong, K. Wang, J.M. Jiang, X.Y. Yu, H.Q. Zhang, Y.D. Guo, Z. Lv, T.J. Gui, An experimental investigation of the composite coating for marine propellers on cavitation characteristics and fouling release property, IOP Conf. Ser. Mater. Sci. Eng. 504 (2019) 012030. https://doi.org/10.1088/1757-899X/504/1/012030.

[39] E. Korkut, M. Atlar, An experimental investigation of the effect of foul release coating application on performance, noise and cavitation characteristics of marine propellers, Ocean Eng. 41 (2012) 1-12. https://doi.org/10.1016/j.oceaneng.2011.12.012.

[40] R. Mutton, M. Atlar, M. Downie, C. Anderson, Drag prevention coatings for marine propellers, in: 2nd Int. Symp. Seawater Drag Reduct., 2005: pp. 23--26.

[41] B. Ji, X. Luo, X. Peng, Y. Wu, H. Xu, Numerical analysis of cavitation evolution and excited 
pressure fluctuation around a propeller in non-uniform wake, Int. J. Multiph. Flow. 43 (2012) 13-21. https://doi.org/10.1016/j.ijmultiphaseflow.2012.02.006.

[42] Y. Long, X. ping Long, B. Ji, W. xin Huai, Z. dong Qian, Verification and validation of URANS simulations of the turbulent cavitating flow around the hydrofoil, J. Hydrodyn. 29 (2017) 610620. https://doi.org/10.1016/S1001-6058(16)60774-6.

[43] Y. Long, X. Long, B. Ji, H. Huang, Numerical simulations of cavitating turbulent flow around a marine propeller behind the hull with analyses of the vorticity distribution and particle tracks, Ocean Eng. 189 (2019) 106310. https://doi.org/10.1016/j.oceaneng.2019.106310.

[44] Y. Long, X. Long, B. Ji, T. Xing, Verification and validation of Large Eddy Simulation of attached cavitating flow around a Clark-Y hydrofoil, Int. J. Multiph. Flow. 115 (2019) 93-107. https://doi.org/10.1016/j.ijmultiphaseflow.2019.03.026.

[45] Y. Long, C. Han, B. Ji, X. Long, Y. Wang, Verification and validation of large eddy simulations of turbulent cavitating flow around two marine propellers with emphasis on the skew angle effects, Appl. Ocean Res. 101 (2020) 102167. https://doi.org/10.1016/j.apor.2020.102167.

[46] Star CCM+14.06, Siemens, User Guid. (2019).

[47] Williams J.E.F., Hawkings D.L., Sound generation by turbulence and surfaces in arbitrary motion, Philos. Trans. R. Soc. London. Ser. A, Math. Phys. Sci. 264 (1969) 321-342. https://doi.org/10.1098/rsta.1969.0031.

[48] Lighthill J.M., On sound generated aerodynamically I. General theory, Proc. R. Soc. London. Ser. A. Math. Phys. Sci. 211 (1952) 564-587. https://doi.org/10.1098/rspa.1952.0060.

[49] Z. Nitzkorski, A novel porous Ffowcs-Williams and Hawkings acoustic methodology for complex geometries, University of the Minnesota, 2015.

[50] F. Farassat, Derivation of Formulations 1 and 1A of Farassat, 2007. NASA/TM-2007-214853.

[51] S. Ianniello, R. Muscari, A. Di Mascio, Ship underwater noise assessment by the acoustic analogy. Part I: Nonlinear analysis of a marine propeller in a uniform flow, J. Mar. Sci. Technol. 18 (2013) 547-570. https://doi.org/10.1007/s00773-013-0227-0.

[52] D.Q. Li, J. Hallander, T. Johansson, Predicting underwater radiated noise of a full scale ship with model testing and numerical methods, Ocean Eng. 161 (2018) 121-135. https://doi.org/10.1016/j.oceaneng.2018.03.027.

[53] P. Di Francescantonio, A new boundary integral formulation for the prediction of sound radiation, J. Sound Vib. 202 (1997) 491-509. https://doi.org/10.1006/jsvi.1996.0843.

[54] M.P. Schultz, The Effect of Biofilms on Turbulent Boundary Layer Structure, PhD Thesis, Florida Institute of Technology, 1998.

[55] K.A. Flack, M.P. Schultz, Review of Hydraulic Roughness Scales in the Fully Rough Regime, J. Fluids Eng. 132 (2010). https://doi.org/10.1115/1.4001492.

[56] M.P. Schultz, G.W. Swain, The influence of biofilms on skin friction drag, Biofouling. 15 (2000) 129-139. https://doi.org/10.1080/08927010009386304.

[57] C. Grigson, Drag Losses of New Ships Caused by Hull Finish, J. Sh. Res. 36 (1992) 182-196.

[58] V.A. Ioselevich, V.N. Pilipenko, Logarithmic velocity profile for flow of a weak polymer solution near a rough surface, SPhD. 18 (1974) 790.

[59] G. Calcagno, F. Di Felice, M. Felli, S. Franchi, F.J.A. Pereira, S. Salvatore, The INSEAN E779a Propeller Test Case: a Database For CFD Validation, 2003.

[60] R.E. Bensow, G. Bark, Implicit LES predictions of the cavitating flow on a propeller, J. Fluids Eng. Trans. ASME. 132 (2010) 0413021-04130210. https://doi.org/10.1115/1.4001342.

[61] C. Yvin, P. Muller, Tip vortex cavitation inception without a cavitation model, in: 19th Numer. Towing Tank Symp. (NuTTS 2016), 2016.

[62] A. Asnaghi, Computational Modelling for Cavitation and Tip Vortex Flows, Chalmers University Of Technology, 2018.

[63] A. Asnaghi, U. Svennberg, R.E. Bensow, Large Eddy Simulations of cavitating tip vortex flows, Ocean Eng. 195 (2020) 106703. https://doi.org/10.1016/j.oceaneng.2019.106703.

[64] G. Kuiper, Cavitation inception on ship propeller models, (1981). 
[65] T. Lloyd, G. Vaz, D. Rijpkema, A. Reverberi, Computational fluid dynamics prediction of marine propeller cavitation including solution verification, 5th International Symposium on Marine Propulsors SMP/17, 2017.

[66] N. Yilmaz, M. Atlar, M. Khorasanchi, An improved Mesh Adaption and Refinement approach to Cavitation Simulation (MARCS) of propellers, Ocean Eng. 171 (2019) 139-150. https://doi.org/10.1016/j.oceaneng.2018.11.001.

[67] ITTC, 27th ITTC Specialist Committee on Hydrodynamic Noise, Noise Discussion Forms., 2014.

[68] V. Viitanen, T. Siikonen, Numerical Simulation Of Cavitating Marine Propeller Flows, in: 9th Natl. Conf. Comput. Mech. MekIT-17, 2017.

[69] S. Gaggero, G. Tani, M. Viviani, F. Conti, A study on the numerical prediction of propellers cavitating tip vortex, Ocean Eng. 92 (2014) 137-161. https://doi.org/10.1016/j.oceaneng.2014.09.042.

[70] ITTC, ITTC 2011. Recommended procedures and guidelines (7.5-03-01-04). CFD, General CFD Verification, (2011).

[71] L.F. Richardson, IX. The approximate arithmetical solution by finite differences of physical problems involving differential equations, with an application to the stresses in a masonry dam, Philos. Trans. R. Soc. London. Ser. A, Contain. Pap. a Math. or Phys. Character. 210 (1911) 307357.

[72] P.J. Roache, Verification of codes and calculations, AIAA J. 36 (1998) 696-702. https://doi.org/10.2514/2.457.

[73] F. Stern, R. Wilson, J. Shao, Quantitative V\&V of CFD simulations and certification of CFD codes, Int. J. Numer. Methods Fluids. 50 (2006) 1335-1355. https://doi.org/10.1002/fld.1090.

[74] C. Celik, D.B. Danisman, P. Kaklis, S. Khan, An investigation into the effect of the Hull Vane on the ship resistance in OpenFOAM, in: Sustain. Dev. Innov. Mar. Technol., CRC Press, 2019: pp. 136-141. https://doi.org/10.1201/9780367810085-17.

[75] V. Krasilnikov, CFD modelling of hydro-acoustic performance of marine propellers: Predicting propeller cavitation, in: Numer. Towing Tank Symp., Portugal, 2019.

[76] R. Muscari, A. Di Mascio, R. Verzicco, Modeling of vortex dynamics in the wake of a marine propeller, Comput. Fluids. 73 (2013) 65-79. https://doi.org/10.1016/j.compfluid.2012.12.003.

[77] E.J. Glover, Propulsive devices for improved propulsive efficiency, Inst. Mar. Eng. Trans. 99 (1987).

[78] ITTC, Specialist Committee on Surface Treatment-Final report and recommendations to the 26th ITTC, Proc. 26th ITTC-Volume II. II (2011) 419-481.

[79] C.-T. Hsiao, G. Chahine, Scaling of Tip Vortex Cavitation Inception for a Marine Open Propeller, 27th Symp. Nav. Hydrodyn. October; Seoul, Korea. (2008).

[80] S.E. Kim, S. Rhee, Toward high-fidelity prediction of tip-vortex around lifting surfaces-what does it take, in: Proc. 25th Symp. Nav. Hydrodyn., 2004: pp. 62-70.

[81] W. Qiu, H. Peng, S. Ni, L. Liu, S. Mintu, D. Hally, C.T. Hsiao, RANS computation of propeller tip vortex flow, Int. J. Offshore Polar Eng. 23 (2013) 73-79.

[82] D.-F. Feder, M. Abdel-Maksoud, Tracking a Tip Vortex with Adaptive Vorticity Confinement and Hybrid RANS-LES, Open J. Fluid Dyn. 06 (2016) 406-429. https://doi.org/10.4236/ojfd.2016.64030.

[83] R.E. Bensow, M. Liefvendahl, An acoustic analogy and scale-resolving flow simulation methodology for the prediction of propeller radiated noise, $31^{\text {st }}$ Symposium on Naval Hydrodynamics, Monterey, CA, USA, 2016.

[84] A.K. Lidtke, T. Lloyd, G. Vaz, Acoustic modelling of a propeller subject to non-uniform inflow | Marin, in: Sixth Int. Symp. Mar. Propulsors, Rome, Italy, 2019.

[85] S. Sezen, M. Atlar, P. Fitzsimmons, N. Sasaki, G. Tani, N. Yilmaz, B. Aktas, Numerical cavitation noise prediction of a benchmark research vessel propeller, Ocean Eng. 211 (2020) 107549. https://doi.org/10.1016/j.oceaneng.2020.107549.

[86] B. Aktas, N. Yilmaz, M. Atlar, N. Sasaki, P. Fitzsimmons, D. Taylor, Suppression of Tip Vortex 
Cavitation Noise of Propellers using PressurePoresTM Technology, J. Mar. Sci. Eng. 8 (2020) 158. https://doi.org/10.3390/jmse8030158.

[87] M. A. Feizi Chekab, P. Ghadimi, S. Reza Djeddi, M. Soroushan, Investigation of Different Methods of Noise Reduction for Submerged Marine Propellers and Their Classification, Am. J. Mech. Eng. 1 (2013) 34-42. https://doi.org/10.12691/ajme-1-2-3.

[88] J. Andersson, D.R. Oliveira, I. Yeginbayeva, M. Leer-Andersen, R.E. Bensow, Review and comparison of methods to model ship hull roughness, Appl. Ocean Res. 99 (2020) 102119. https://doi.org/10.1016/j.apor.2020.102119. 